INSTITUT NATIONAL DE LA STATISTIQUE ET DES ETUDES ECONOMIQUES

Série des Documents de Travail du CREST

(Centre de Recherche en Economie et Statistique)

\title{
$n^{\circ}$ 2007-19
}

\section{Switching VARMA Term Structure Models Extended Version}

\author{
A. MONFORT ${ }_{1}$ \\ F. PEGORARO
}

Les documents de travail ne reflètent pas la position de l'INSEE et n'engagent que leurs auteurs.

Working papers do not reflect the position of INSEE but only the views of the authors.

${ }^{1}$ CNAM and CREST, Laboratoire de Finance-Assurance. (monfort@cnam.fr and monfort@ensae.fr)

2 Banque de France, Economics and Finance Research Center [DGEI-DIR-RECFIN] and CREST, Laboratoire de Finance-Assurance (fulvio.pegoraro@banque-france.fr and pegoraro@ensae.fr) 


\title{
Switching VARMA Term Structure Models Extended Version
}

\author{
Alain MONFORT ${ }^{(1)}$ Fulvio PEGORARO ${ }^{(2)}$
}

This version : November, 2006

\author{
Abstract \\ Switching VARMA Term Structure Models \\ Extended Version
}

\begin{abstract}
The purpose of the paper is to propose a global discrete-time modeling of the term structure of interest rates able to capture simultaneously the following important features : (i) an historical dynamics of the factor driving term structure shapes involving several lagged values, and switching regimes; (ii) a specification of the stochastic discount factor (SDF) with time-varying and regime-dependent risk-premia; (iii) explicit or quasi explicit formulas for zero-coupon bond and interest rate derivative prices; (iv) the positivity of the yields at each maturity. The first family of models we develop is given by the Switching Autoregressive Normal (SARN) and the Switching Vector Autoregressive Normal (SVARN) Factor-Based Term Structure Models of order $p$. The second family of models we study is given by the Switching Autoregressive Gamma (SARG) and the Switching Vector Autoregressive Gamma (SVARG) Factor-Based Term Structure Models of order $p$. Regime shifts are described by a Markov chain with (historical) non-homogeneous transition probabilities.
\end{abstract}

Keywords : Affine Term Structure Models, Stochastic Discount Factor, Car processes, Switching Regimes, VARMA processes, Lags, Positivity, Derivative Pricing.

\section{Résumé \\ Switching VARMA Term Structure Models Extended Version}

Le but de ce papier est de proposer une modélisation globale en temps discret de la courbe de taux d'intérêt capable de capturer simultanément les aspect suivants : (i) une dynamique historique du facteur déterminant la courbe de taux caractérisée par des retards et des changements de régimes; (ii) une spécification du facteur d'escompte stochastique avec des coefficients d'ajustement pour le risque stochastiques et dépendant de régimes; (iii) des formules de prix de zero-coupons et de dérivés sur taux sous une forme explicite ou quasi explicite; (iv) des taux positifs pour toute maturité. La première famille de modèles est constituée des Switching Autoregressive Normal (SARN) et des Switching Vector Autoregressive Normal (SVARN) modèles à facteurs pour la structure par terme des taux d'intérêt. La deuxième famille de modèles contient les Switching Autoregressive Gamma (SARG) et les Switching Vector Autoregressive Gamma (SVARG) modèles à facteurs pour la structure par terme des taux d'intérêt. Les changements de régimes sont décrits par une chaîne de Markov non-homogène.

Mots Clés : Modèles Affines pour la courbe de taux d'intérêt, facteur d'escompte stochastique, processus Car, Changement de Régimes, processus VARMA, Lags, Positivité, Valorisation de Dérivés.

JEL number : C1, C5, E43, G12

\footnotetext{
${ }^{1}$ CNAM [E-mail: monfort@cnam.fr] and CREST, Laboratoire de Finance-Assurance [E-mail: monfort@ensae.fr]. ${ }^{2}$ Banque de France, Economics and Finance Research Center [DGEI-DIR-RECFIN; E-mail: Fulvio.PEGORARO@banque-france.fr] and CREST, Laboratoire de Finance-Assurance [E-mail: pegoraro@ensae.fr].
} 


\section{Introduction}

In this paper we propose a global discrete-time modeling of the term structure of interest rates, which captures simultaneously the following important features :

- an historical dynamics of the factor driving term structure shapes involving several lagged values, and switching regimes;

- a specification of the stochastic discount factor (SDF) with time-varying and regime-dependent risk-premia;

- explicit or quasi explicit formulas for zero-coupon bond and interest rate derivative prices;

- the positivity of the yields at each maturity.

It is well known in the literature that interest rates show an historical dynamics involving lagged values and switching regimes [see, among the others, Hamilton (1988), Cai (1994), Driffill and Sola (1994), Garcia and Perron (1996), Gray (1996), Boudoukh, Richardson, Smith, and Whitelaw (1999), Ang and Bekaert (2002a, 2002b), Christiansen (2004), Christiansen and Lund (2005), Cochrane and Piazzesi (2005)]; indeed, changes in the business cycle conditions or monetary policy may affect real rates and expected inflation and cause interest rates to behave quite differently in different time periods, both in terms of level and volatility. In addition, there is a large empirical literature on bond yields, based in general on the class of Affine Term Structure Models (ATSMs) ${ }^{3}$, suggesting that regime switching models describe the term structure of interest rates better than single-regime models [see, for example, Bansal and Zhou (2002), Driffill, Kenc and Sola (2003), Evans (2003), Ang and Bekaert (2005), Dai Singleton and Yang (2006)].

These results lead us to propose dynamic term structure models (DTSMs) where the yield curve is driven by a univariate or multivariate factor $\left(x_{t}\right)$ which depends on its $p$ most recent lagged values $\left[X_{t}\right.$, say] and for which all the coefficients depend on the present and past values of a latent $J$-state non homogeneous Markov Chain $\left(z_{t}\right)\left[Z_{t}\right.$, say] describing different regimes in the economy. Consequently, the joint dynamics of $\left(x_{t}, z_{t}\right)$ is not a Compound Autoregressive (Car) process ${ }^{4}$ under the historical probability, and thus allows for nonlinearities already documented by the literature [see Ait-Sahalia (1996), Stanton (1997), Ang and Bekaert (2002b)]. The factor $\left(x_{t}\right)$ is considered as a latent variable or an observable variable: in the second case the factor is a vector of several yields.

We consider an exponential-affine SDF with time-varying and regime-dependent risk correction coefficients which are defined as functions of the present and past values of the factor $\left(x_{t}\right)$ and of the regime indicator function $\left(z_{t}\right)$. In our models, both factor risk and regime-shift risk are priced, and this is done by taking into account not just the information at date $t$, that is $\left(x_{t}, z_{t}\right)$, but a larger information given by $\left(X_{t}, Z_{t}\right)$. This specification leads to stochastic and regime-dependent

\footnotetext{
${ }^{3}$ The Affine family of dynamic term structure models (DTSMs) is characterized by the fact that the zero-coupon bond yields are affine functions of Markovian state variables, and it gives closed-form expressions for zero-coupon bond prices which greatly facilitates pricing and econometric implementation [see Vasicek (1977), Duffie and Kan (1996), Dai and Singleton (2000, 2003) and Piazzesi (2003)]. The Affine Term Structure family is much larger that it has been considered in the literature : indeed, it has been observed recently that the family of Quadratic Term Structure Models (QTSMs) [see Beaglehole and Tenney (1991), Ahn, Dittmar and Gallant (2002), and Leippold and $\mathrm{Wu}(2002)]$ is a special case of the Affine class obtained by stacking the factor values and their squares [see Gourieroux and Sufana (2003), Cheng and Scaillet (2005)].

${ }^{4} \mathrm{~A}$ Car (discrete-time affine) process is a Markovian process with an exponential-affine conditional Laplace transform [see Darolles, Gourieroux, Jasiak (2006) for details].
} 
risk premia and is coherent with recent empirical literature suggesting to define risk correction coefficients as functions of both factors and their volatilities. Such a specification is helpful in order to replicate correctly the observed temporal variation of one-period expected excess returns on zero-coupon bonds [see Ahn, Dittmar and Gallant (2002), Dai and Singleton (2002), Duffee (2002), Duarte (2004), Cheridito, Filipovic and Kimmel (2005), Dai, Singleton and Yang (2006)].

At the same time, we want to exploit the tractability of Car models, and obtain explicit or quasi explicit formula for zero-coupon bond and interest rate derivative prices. This result is achieved by matching the historical distribution and the SDF in order to get a Car risk-neutral joint dynamics for $\left(x_{t}, z_{t}\right)$, and by using the property of the Car family of being able to incorporate lags and switching regimes. It is now well known [see Gourieroux, Monfort and Polimenis (2006), and Darolles, Gourieroux, Jasiak (2006)] that the class of discrete-time affine (Car) models is much larger than the discrete-time counterparts of the continuous-time affine processes [see Duffie and Kan (1996), Dai and Singleton (2000), and Duffie, Filipovic and Schachermayer (2003)].

We develop the Switching Autoregressive Normal (SARN) and the Switching Vector Autoregressive Normal (SVARN) Factor-Based Term Structure Models of order $p$. Ang and Bekaert (2005) also propose a discrete time regime-switching Gaussian term structure model (to identify the real and expected inflation components of nominal interest rates). In their model, the historical dynamics of the tridimensional factor $\left(x_{t}\right)$ driving term structure shapes is described by a regimeswitching $\operatorname{VAR}(1)$ process with a constant autoregressive matrix. The regime indicator function $\left(z_{t}\right)$ is driven, under the historical probability, by a homogeneous Markov chain and regime-shift risk is not priced. Bansal and Zhou (2002) propose a bivariate (approximate) discrete-time CoxIngersoll-Ross term structure model with regime shifts. In their modeling, $\left(z_{t}\right)$ is a homogeneous Markov chain under the historical probability; the associated risk correction coefficient is assumed equal to zero, and the provided term structure formula is based on a log-linear approximation applied on the fundamental asset pricing equation. Our SVARN $(p)$ Factor-Based Term Structure Model relax all these assumptions.

Dai, Singleton and Yang (2006) propose a Gaussian discrete time model where the historical dynamics of the latent factor $\left(x_{t}\right)$ is described by a trivariate SVARN(1) process with nonhomogeneous regime-switching. They price regime-shift risk, and their factor risk correction coefficient generalizes to the case of multiple regimes the essentially affine specification of Duffee (2002). In our approach, the historical dynamics of $\left(x_{t}\right)$ depends on several lagged values and on several past non-homogeneous regime-indicators $\left(z_{t}\right)$ [the $\operatorname{SVARN}(p)$ process], we price regime-shift risk and our specification of the factor risk correction coefficient extends to the case of multiple lags that of Dai, Singleton and Yang (2006). Moreover, in the empirical analysis of $\operatorname{SVARN}(p)$ FactorBased Term Structure Models, we overcome their identification problems given that the factor $\left(x_{t}\right)$ will be observable (yields at different maturities). In this general setting, we are able to derive formulas, as well as for the yield curve and for the price of derivatives, with simple analytical or quasi explicit representations.

The second famity of models we study in the paper, based on the (scalar and vector) Switching Autoregressive Gamma process ${ }^{5}$ of order $p$, implies the positivity of the yields for each time to maturity, and regardless the latent or observable nature of the factor $\left(x_{t}\right)$. The Switching Autoregressive Gamma (SARG) and the the Switching Vector Autoregressive Gamma (SVARG) Factor-Based Term Structure Models of order $p$ give the possibility to replicate complex nonlinear (historical and risk-neutral) factor dynamics and provide explicit or tractable formulas for zero-coupon bond and derivative prices. In a related study, Bansal and Zhou (2002) propose an

\footnotetext{
${ }^{5}$ The Autoregressive Gamma (ARG) process is a Car process, and the ARG(1) specification is the discrete-time counterpart of the Cox-Ingersoll-Ross process [see Gourieroux and Jasiak (2006), Cox, Ingersoll, and Ross (1985)].
} 
(approximate, scalar and bivariate) discrete-time Cox-Ingersoll-Ross term structure model with regime shifts. We extend the Bansal and Zhou (2002) framework in several directions; we use the exact discrete-time equivalent of the CIR process (with switching regimes) generalized to an autoregressive order $p$ larger than one; we allow for a non-homogeneous historical transition matrix for $\left(z_{t}\right)$; we price the regime-shift risk, and we provide an exact yield-to-maturity formula [in Bansal and Zhou (2002), $\left(z_{t}\right)$ is an homogeneous Markov chain, the associated risk correction coefficient is assumed equal to zero, and the term structure formula they provide is based on a log-linear approximation applied on the fundamental asset pricing equation].

In a recent paper Dai, Le and Singleton (2006) propose a (discrete-time multivariate) conditionally Gaussian term structure model with stochastic volatility. Under the risk-neutral probability, the (multivariate) stochastic volatility factor is described by a particular VARG(1) process with conditionally independent components. The switching vector Autoregressive Gamma process we use to describe the risk-neutral dynamics of the factor $\left(x_{t}\right)$, in the SVARG $(p)$ Factor-Based Term Structure Model, presents three generalizations with respect to their specification: a) we consider an autoregressive order $p$ in general larger than one; b) conditionally to the present and past values of $x_{t}$ and $z_{t}$, there is dependence between the components of the factor $x_{t+1}$; c) the historical and risk-neutral dynamics of $x_{t+1}$ is affected by switching regimes.

The plan of the paper is as follows. In Section 2, we present the Index-Car $(p)$ processes. This family of processes is developed in a univariate and multivariate setting, with and without Switching Regimes. In particular, we study the (scalar and vector) Autoregressive Gaussian of order $p$ models and the (scalar and vector) Autoregressive Gamma of order $p$ models, under single-regime and regime-switching specifications. Then, this class of processes is used, following the SDF modeling principle, to the derive the $\operatorname{SARN}(p)$ and the $\operatorname{SARG}(p)$ Factor-Based Term Structure Models, and their multivariate generalizations. In Section 3 we study the $\operatorname{SARN}(p)$ and the $\operatorname{SVARN}(p)$ specifications, we derive the Generalized Linear Term Structure formulas and we specify the historical and risk-neutral dynamics of the yield curve processes. These results are given for a latent or an observable factor. We discuss the propagation of shocks on the interest rate surface, and we present a two-step estimation procedure for a $\operatorname{SVARN}(p)$ Factor-Based Term Structure Model with observable factor. The second step of this estimation methodology is based on a generalization of the Kim's smoothing algorithm. Section 4 deal with the SARG $(p)$ and the SVARG $(p)$ FactorBased Term Structure Models. Here, regardless the observable or latent nature of the factor $\left(x_{t}\right)$, we derive the Generalized Linear Term Structure formulas and the yield curve processes, and we guarantee the positivity of the yields for each time to maturity. Then, the pricing methodology proposed in Sections 3 and 4, for zero-coupon bonds, is generalized in Section 5 to the case of interest rate derivatives. Section 6 concludes and appendices gather the proofs.

\section{Laplace Transforms, $\operatorname{Car}(p)$ Processes and Switching Regimes}

It is now well documented [see e.g. Darolles, Gourieroux and Jasiak (2006), Gourieroux and Jasiak (2006), Gourieroux, Jasiak and Sufana (2004), Gourieroux and Monfort (2006a), Gourieroux, Monfort and Polimenis (2003, 2006), Pegoraro (2006), Polimenis (2001)] that the Laplace transform (or moment generating function) is a very convenient mathematical tool in many financial domains. It is, in particular, a crucial notion in the theory of Car $(p)$ processes [see Darolles, Gourieroux and Jasiak (2006) for details]. 


\subsection{Definition of a $\operatorname{Car}(p)$ process}

Definition $1\left[\operatorname{Car}(p)\right.$ process]: A $n$-dimensional process $\tilde{x}=\left(\tilde{x}_{t}, t \geq 0\right)$ is a compound autoregressive process of order $p[\operatorname{Car}(p)]$ if the distribution of $\tilde{x}_{t+1}$ given the past values $\underline{\tilde{x}_{t}}=\left(\tilde{x}_{t}, \tilde{x}_{t-1}, \ldots\right)$ admits a real Laplace transform of the following type:

$$
\begin{aligned}
E\left[\exp \left(u^{\prime} \tilde{x}_{t+1}\right) \mid \underline{\left.\tilde{x}_{t}\right]}\right. & =E_{t}\left[\exp \left(u^{\prime} \tilde{x}_{t+1}\right)\right] \\
& =\exp \left[\tilde{a}_{1}(u)^{\prime} \tilde{x}_{t}+\ldots+\tilde{a}_{p}(u)^{\prime} \tilde{x}_{t+1-p}+\tilde{b}(u)\right], \quad u \in \mathbb{R}^{n},
\end{aligned}
$$

where $a_{i}(u), i \in\{1, \ldots, p\}$, and $b(u)$ are nonlinear functions, and where $a_{p}(u) \neq 0, \forall u \in \mathbb{R}^{n}$. The existence of this Laplace transform in a neighborhood of $u=0$, implies that all the conditional moments exist, and that the conditional expectations and variance-covariance matrices (and all conditional cumulants) are affine functions of $\left(\tilde{x}_{t}^{\prime}, \tilde{x}_{t-1}^{\prime}, \ldots, \tilde{x}_{t+1-p}^{\prime}\right)$.

\subsection{Univariate Index-Car $(p)$ process}

An important class of $\operatorname{Car}(p)$ processes are the Index-Car $(p)$ processes, which are built from a Car(1) process. In this section we consider a univariate process $x_{t}$ and the multivariate case will be considered in Sections 2.6 and 2.7.

Definition 2 [Univariate Index-Car $(p)$ process]: Let $\exp \left[a(u) y_{t}+b(u)\right]$ be the conditional Laplace transform of a univariate Car(1) process $y_{t}$, the process $x_{t}$ admitting a conditional Laplace transform defined by:

$$
E\left[\exp \left(u x_{t+1}\right) \mid \underline{x_{t}}\right]=\exp \left[a(u)\left(\beta_{1} x_{t}+\ldots+\beta_{p} x_{t+1-p}\right)+b(u)\right], u \in \mathbb{R},
$$

is called an Univariate Index-Car $(p)$ process.

Note that, if $y_{t}$ is a positive process and if the parameters $\beta_{1}, \ldots, \beta_{p}$ are positive, the process $x_{t}$ will be positive.

Using the notation $\beta=\left(\beta_{1}, \ldots, \beta_{p}\right)^{\prime}$ and $X_{t}=\left(x_{t}, x_{t-1}, \ldots, x_{t+1-p}\right)^{\prime}$, the Laplace transform (2) can be written as:

$$
E\left[\exp \left(u x_{t+1}\right) \mid \underline{x_{t}}\right]=\exp \left[a(u) \beta^{\prime} X_{t}+b(u)\right] .
$$

\subsection{Examples of Univariate Index-Car $(p)$ processes}

\section{a. Gaussian model}

If $y_{t}$ is a Gaussian $\mathrm{AR}(1)$ process defined by:

$$
y_{t+1}=\nu+\rho y_{t}+\varepsilon_{t+1}
$$

where $\varepsilon_{t+1}$ is a gaussian white noise distributed as $\mathcal{N}\left(0, \sigma^{2}\right)$, the conditional Laplace transform of $y_{t+1}$ given $y_{t}$ is:

$$
E\left[\exp \left(u y_{t+1}\right) \mid \underline{y_{t}}\right]=\exp \left[u \rho y_{t}+u \nu+\frac{\sigma^{2}}{2} u^{2}\right] .
$$

The process is Car $(1)$ with $a(u)=u \rho$ and $b(u)=u \nu+\frac{\sigma^{2}}{2} u^{2}$. The associated Index-Car $(p)$ process has a conditional Laplace transform defined by:

$$
E\left[\exp \left(u x_{t+1}\right) \mid \underline{x_{t}}\right]=\exp \left[u \rho\left(\beta_{1} x_{t}+\ldots+\beta_{p} x_{t+1-p}\right)+u \nu+\frac{\sigma^{2}}{2} u^{2}\right]
$$


so, using the notation $\varphi_{i}=\rho \beta_{i}$, we see that $x_{t+1}$ is the Gaussian $\operatorname{AR}(p)$ process defined by:

$$
x_{t+1}=\nu+\varphi_{1} x_{t}+\ldots+\varphi_{p} x_{t+1-p}+\varepsilon_{t+1}
$$

and its conditional Laplace transform becomes:

$$
E\left[\exp \left(u x_{t+1}\right) \mid \underline{x_{t}}\right]=\exp \left[u \varphi^{\prime} X_{t}+u \nu+\frac{\sigma^{2}}{2} u^{2}\right],
$$

where $\varphi=\left(\varphi_{1}, \ldots, \varphi_{p}\right)^{\prime}$.

\section{b. Gamma model}

Let us now consider an autoregressive gamma of order one $[\mathrm{ARG}(1)]$ process $y_{t}$. The conditional Laplace transform is [see Gourieroux and Jasiak (2005) for details]:

$$
E\left[\exp \left(u y_{t+1}\right) \mid \underline{y_{t}}\right]=\exp \left[\frac{\rho u}{1-u \mu} y_{t}-\nu \log (1-u \mu)\right], \rho>0, \mu>0, \nu>0,
$$

and it is well known that, given $y_{t}, y_{t+1}$ can be obtained by first drawing a latent variable $U_{t+1}$ in the Poisson distribution $\mathcal{P}\left(\frac{\rho y_{t}}{\mu}\right)$ and, then, drawing $\frac{y_{t+1}}{\mu}$ in the gamma distribution $\gamma\left(\nu+U_{t+1}\right)$. The process $y_{t+1}$ is positive and the associated Index-Car $(p)$ process $x_{t+1}$ is also positive. The conditional Laplace transform of this process is:

$$
E\left[\exp \left(u x_{t+1}\right) \mid \underline{x_{t}}\right]=\exp \left[\frac{\rho u}{1-u \mu}\left(\beta_{1} x_{t}+\ldots+\beta_{p} x_{t+1-p}\right)-\nu \log (1-u \mu)\right],
$$

with $\beta_{i} \geq 0$, for $i \in\{1, \ldots, p\}$, or using the same notation as above:

$$
E\left[\exp \left(u x_{t+1}\right) \mid \underline{x_{t}}\right]=\exp \left[\frac{u}{1-u \mu} \varphi^{\prime} X_{t}-\nu \log (1-u \mu)\right] .
$$

Similarly, given $X_{t}, x_{t+1}$ can be obtained by drawing $U_{t+1}$ in $\mathcal{P}\left(\frac{\varphi^{\prime} X_{t}}{\mu}\right)$ and $\frac{x_{t+1}}{\mu}$ in $\gamma\left(\nu+U_{t+1}\right)$. It easily seen that the conditional mean and variance of $x_{t+1}$, given $x_{t}$, are respectively given by $\nu \mu+\varphi^{\prime} X_{t}$ and $\nu \mu^{2}+2 \mu \varphi^{\prime} X_{t} ;$ so, the process $x_{t+1}$ has the weak $\operatorname{AR}(p)$ representation:

$$
x_{t+1}=\nu \mu+\varphi^{\prime} X_{t}+\varepsilon_{t+1},
$$

where $\varepsilon_{t+1}$ is a conditionally heteroscedastic martingale difference, whose conditional variance is $\nu \mu^{2}+2 \mu \varphi^{\prime} X_{t}$; the process is stationary if and only if $\varphi^{\prime} e<1\left[\right.$ where $\left.e=(1, \ldots, 1) \in \mathbb{R}^{p}\right]$ and,

in this case, the process $\varepsilon_{t+1}$ has finite unconditional variance given by $\nu \mu^{2}+2 \nu \mu^{2} \frac{\varphi^{\prime} e}{1-\varphi^{\prime} e}$. The unconditional mean of $x_{t+1}$ is given by $\frac{\nu \mu}{1-\varphi^{\prime} e}$.

\subsection{Univariate Switching regimes $\operatorname{Car}(p)$ process}

Let us first consider a $J$-state homogeneous Markov Chain $z_{t+1}$, which can take the values $e_{j} \in \mathbb{R}^{J}$, $j \in\{1, \ldots, J\}$, where $e_{j}$ is the $j^{t h}$ column of the $(J \times J)$ identity matrix. The transition probability, from state $e_{i}$ to state $e_{j}$ is $\pi\left(e_{i}, e_{j}\right)=\operatorname{Pr}\left(z_{t+1}=e_{j} \mid z_{t}=e_{i}\right)$. It is first worth noting that $z_{t+1}$ is a Car(1) process.

Proposition 1 : The Markov chain process $z_{t+1}$ is a Car(1) process with a conditional Laplace transform given by:

$$
E\left[\exp \left(v^{\prime} z_{t+1}\right) \mid \underline{z_{t}}\right]=\exp \left(a_{z}(v, \pi)^{\prime} z_{t}\right)
$$


where

$$
a_{z}(v, \pi)=\left[\log \left(\sum_{j=1}^{J} \exp \left(v^{\prime} e_{j}\right) \pi\left(e_{1}, e_{j}\right)\right), \ldots, \log \left(\sum_{j=1}^{J} \exp \left(v^{\prime} e_{j}\right) \pi\left(e_{J}, e_{j}\right)\right)\right]^{\prime}
$$

[Proof : straightforward].

Let us now consider a univariate Index-Car $(p)$ process with a conditional Laplace transform given by $\exp \left[a(u) \beta^{\prime} X_{t}+b(u)\right]$, and let us assume that $b(u)$ can be written:

$$
\begin{aligned}
b(u) & =\tilde{b}(u)^{\prime} \lambda \text { where } \\
\tilde{b}(u) & =\left(b_{1}(u), \ldots, b_{m}(u)\right)^{\prime} \text { and } \lambda=\left(\lambda_{1}, \ldots, \lambda_{m}\right)^{\prime} .
\end{aligned}
$$

We can generalize this model by assuming that the parameters $\lambda_{i}$ are stochastic and linear functions of $Z_{t}=\left(z_{t}^{\prime}, \ldots, z_{t-p}^{\prime}\right)^{\prime}$. More precisely, we assume that the conditional distribution of $x_{t+1}$ given $\underline{x_{t}}$ and $\underline{z_{t+1}}$ has a Laplace transform given by:

$$
E\left[\exp \left(u x_{t+1}\right) \mid \underline{x_{t}}, \underline{z_{t+1}}\right]=\exp \left[a(u) \beta^{\prime} X_{t}+\tilde{b}(u)^{\prime} \Lambda Z_{t}\right],
$$

where $\Lambda$ is a $[m,(p+1) J]$ matrix. Note that we assume no instantaneous causality between $x_{t+1}$ and $z_{t+1}$ and we admit one more lag in $Z_{t}$ that in $X_{t}$ [examples given in Section 2.5 show that this assumption may be convenient]; if the process $z_{t}$ is not observed by the econometrician the no instantaneous causality assumption is not really important at the estimation stage since we could rename $z_{t}$ as $z_{t+1}$, however it will be useful at the pricing level in order to obtain simple pricing procedures [Dai, Singleton and Yang (2006) also make this kind of assumption]. The joint process $\left(x_{t+1}, z_{t+1}^{\prime}\right)^{\prime}$ is easily seen to be a $\operatorname{Car}(p+1)$ process.

Proposition 2 : The conditional Laplace transform of $\left(x_{t+1}, z_{t+1}^{\prime}\right)^{\prime}$ given $\underline{x_{t}}, \underline{z_{t}}$ has the following form:

$$
E\left[\exp \left(u x_{t+1}+v^{\prime} z_{t+1}\right) \mid \underline{z_{t}}, \underline{x_{t}}\right]=\exp \left\{a(u) \beta^{\prime} X_{t}+\left[e_{1}^{\prime} \otimes a_{z}(v, \pi)^{\prime}+\tilde{b}(u)^{\prime} \Lambda\right] Z_{t}\right\}
$$

where $e_{1}$ is the first component of the canonical basis in $\mathbb{R}^{p+1}$, and where $\otimes$ denotes the Kronecker product [Proof : straightforward].

\subsection{Examples of Univariate Switching regimes Car $(p)$ processes}

\section{a. Gaussian case}

Let us start from the $\operatorname{AR}(p)$ model (4). Its conditional Laplace transform is given by (5):

$$
E\left[\exp \left(u x_{t+1}\right) \mid \underline{x_{t}}\right]=\exp \left[u \varphi^{\prime} X_{t}+u \nu+\frac{\sigma^{2}}{2} u^{2}\right]
$$

and the function $b(u)$ has the form $(9)$ with $\tilde{b}(u)^{\prime}=\left(u, \frac{u^{2}}{2}\right)$ and $\lambda^{\prime}=\left(\nu, \sigma^{2}\right)$.

If $\lambda$ is replaced by $\Lambda Z_{t}$, the joint process $\left(x_{t+1}, z_{t+1}^{\prime}\right)^{\prime}$ is $\operatorname{Car}(p+1)$ with a conditional Laplace transform given by:

$$
E\left[\exp \left(u x_{t+1}+v^{\prime} z_{t+1}\right) \mid \underline{z_{t}}, \underline{x_{t}}\right]=\exp \left[u \varphi^{\prime} X_{t}+\left(u, \frac{u^{2}}{2}\right) \Lambda Z_{t}+a_{z}(v, \pi) z_{t}\right] .
$$


More precisely, the dynamics is given by [using the notation $\Lambda=\left(\begin{array}{c}\lambda_{1}^{\prime} \\ \lambda_{2}^{\prime}\end{array}\right)$ ]:

$$
x_{t+1}=\lambda_{1}^{\prime} Z_{t}+\varphi^{\prime} X_{t}+\left(\lambda_{2}^{\prime} Z_{t}\right)^{1 / 2} \varepsilon_{t+1},
$$

where $\varepsilon_{t+1}$ is a gaussian white noise distributed as $\mathcal{N}\left(0, \sigma^{2}\right), Z_{t}=\left(z_{t}^{\prime}, \ldots, z_{t-p}^{\prime}\right)^{\prime}$ and $z_{t}$ is a Markov chain such that $\operatorname{Pr}\left(z_{t+1}=e_{j} \mid z_{t}=e_{i}\right)=\pi\left(e_{i}, e_{j}\right)$.

In particular, let us consider the case:

$$
\Lambda=\left[\begin{array}{c}
\left(1,-\varphi_{1}, \ldots,-\varphi_{p}\right) \otimes \nu^{*^{\prime}} \\
e_{1}^{\prime} \otimes \sigma^{* 2^{\prime}}
\end{array}\right]
$$

and $\nu^{*^{\prime}}=\left(\nu_{1}^{*}, \ldots, \nu_{J}^{*}\right), \sigma^{* 2^{\prime}}=\left(\sigma_{1}^{* 2}, \ldots, \sigma_{J}^{* 2}\right)$, the conditional distribution of $x_{t+1}$ given $\underline{x_{t}}$ and $\underline{z_{t+1}}$ is the one corresponding to the switching $\operatorname{AR}(p)$ model defined by:

$$
x_{t+1}-\nu^{*^{\prime}} z_{t}=\varphi_{1}\left(x_{t}-\nu^{*^{\prime}} z_{t-1}\right)+\ldots+\varphi_{p}\left(x_{t+1-p}-\nu^{*^{\prime}} z_{t-p}\right)+\left(\sigma^{*^{\prime}} z_{t}\right) \varepsilon_{t+1} .
$$

\section{b. Gamma case}

Let us now start from the $\operatorname{ARG}(p)$ process associated with the conditional Laplace transform (6):

$$
E\left[\exp \left(u x_{t+1}\right) \mid \underline{x_{t}}\right]=\exp \left[\frac{u}{1-u \mu} \varphi^{\prime} X_{t}-\nu \log (1-u \mu)\right] .
$$

Here we have $\tilde{b}(u)=-\log (1-u \mu)$ and $\lambda=\nu$. If $\nu$ is replaced by $\Lambda Z_{t}$, where $\Lambda Z_{t}>0$, the process $x_{t}$ has, conditionally to the process $z_{t}$, a weak $\operatorname{AR}(p)$ representation given by:

$$
x_{t+1}=\mu \Lambda Z_{t}+\varphi_{1} x_{t}+\ldots+\varphi_{p} x_{t+1-p}+\zeta_{t+1},
$$

where $\zeta_{t+1}$ is a conditionally heteroscedastic martingale difference. For instance, we can take :

$$
\Lambda=e_{1}^{\prime} \otimes \frac{\tilde{\nu}^{\prime}}{\mu}
$$

where $\tilde{\nu}^{\prime}=\left(\tilde{\nu}_{1}, \ldots, \tilde{\nu}_{J}\right), \tilde{\nu}_{j} \geq 0$. We have $\Lambda Z_{t}=\frac{\tilde{\nu}^{\prime}}{\mu} z_{t}$ and, conditionally to the process $z_{t}$, the process $x_{t}$ has a weak $\operatorname{AR}(p)$ representation given by:

$$
x_{t+1}=\tilde{\nu}^{\prime} z_{t}+\varphi_{1} x_{t}+\ldots+\varphi_{p} x_{t+1-p}+\zeta_{t+1} .
$$

It is also possible to consider a $\Lambda$ of the form $\left(1,-\varphi_{1}, \ldots,-\varphi_{p}\right) \otimes \frac{\tilde{\nu}^{\prime}}{\mu}$ if $\min \left(\tilde{\nu}_{i}\right)>\max \left(\tilde{\nu}_{i}\right) \sum_{i=1}^{J} \varphi_{j}$, since in this case $\Lambda Z_{t}=\frac{1}{\mu}\left(\tilde{\nu}^{\prime} z_{t}-\sum_{i=1}^{J} \varphi_{j} \tilde{\nu}^{\prime} z_{t-i}\right) \geq 0$. The weak conditional $\operatorname{AR}(p)$ representation is then given by:

$$
x_{t+1}-\tilde{\nu}^{\prime} z_{t}=\varphi_{1}\left(x_{t}-\tilde{\nu}^{\prime} z_{t-1}\right)+\ldots+\varphi_{p}\left(x_{t+1-p}-\tilde{\nu}^{\prime} z_{t-p}\right)+\zeta_{t+1} .
$$

\subsection{Specification of multivariate $\operatorname{Car}(1)$ processes}

In order to have simple notations we will consider the bivariate case, but all the results are easily extended to the general case. A bivariate Car(1) process $y_{t}=\left(y_{1, t}, y_{2, t}\right)^{\prime}$ will be defined in a recursive way. We consider two univariate exponential affine Laplace transforms :

$$
\begin{aligned}
& \exp \left[a_{1}\left(u_{1}\right) w_{1, t}+b_{1}\left(u_{1}\right)\right], \\
\text { and } \quad & \exp \left[a_{2}\left(u_{2}\right) w_{2, t}+b_{2}\left(u_{2}\right)\right] .
\end{aligned}
$$


Then, we assume that the conditional distribution of $y_{1, t+1}$ given $\left(y_{2, t+1}, \underline{y_{1, t}}, \underline{y_{2, t}}\right)$ has a Laplace transform given by :

$$
E_{t}\left[\exp \left(u_{1} y_{1, t+1}\right) \mid y_{2, t+1}, \underline{y_{1 t}}, \underline{y_{2 t}}\right]=\exp \left[a_{1}\left(u_{1}\right)\left(\beta_{o} y_{2, t+1}+\beta_{11} y_{1, t}+\beta_{12} y_{2, t}\right)+b_{1}\left(u_{1}\right)\right]
$$

and the conditional distribution of $y_{2, t+1}$, given $\left(\underline{y_{1, t}}, \underline{y_{2, t}}\right)$, has a Laplace transform given by

$$
E_{t}\left[\exp \left(u_{2} y_{2, t+1}\right) \mid \underline{y_{1, t}}, \underline{y_{2, t}}\right]=\exp \left[a_{2}\left(u_{2}\right)\left(\beta_{21} y_{1, t}+\beta_{22} y_{2, t}\right)+b_{2}\left(u_{2}\right)\right]
$$

Note that, if the Laplace transforms (20) correspond to positive variables and if the parameters $\beta_{o}, \beta_{11}, \beta_{12}, \beta_{21}, \beta_{22}$ are positive the bivariate process $y_{t}$ has positive components. Moreover, we have the following result :

Proposition 3 : The bivariate process $y_{t}$ defined by the conditional dynamics $(21),(22)$ is a bivariate Car(1) process with a conditional Laplace transform given by :

$$
\begin{aligned}
E\left[\exp \left(u_{1} y_{1, t+1}+u_{2} y_{2, t+1}\right) \mid \underline{y_{1, t}}, \underline{\left.y_{2, t}\right]}=\exp \{\right. & {\left[a_{1}\left(u_{1}\right) \beta_{11}+a_{2}\left(u_{2}+a_{1}\left(u_{1}\right) \beta_{o}\right) \beta_{21}\right] y_{1, t} } \\
& +\left[a_{1}\left(u_{1}\right) \beta_{12}+a_{2}\left(u_{2}+a_{1}\left(u_{1}\right) \beta_{o}\right) \beta_{22}\right] y_{2, t} \\
& \left.+b_{1}\left(u_{1}\right)+b_{2}\left(u_{2}+a_{1}\left(u_{1}\right) \beta_{o}\right)\right\}
\end{aligned}
$$

[Proof : see Appendix 1].

\subsection{Specification of multivariate Index-Car $(p)$ processes}

We consider a bivariate process $\tilde{x}_{t}=\left(x_{1, t}, x_{2, t}\right)^{\prime}$ and we introduce the notations : $X_{1 t}=\left(x_{1, t}, \ldots\right.$, $\left.x_{1, t+1-p}\right)^{\prime}, X_{2 t}=\left(x_{2, t}, \ldots, x_{2, t+1-p}\right)^{\prime}$. Given the univariate Laplace transforms like (20), a bivariate Index-Car $(p)$ is defined in the following way.

Definition 3 : A bivariate Index-Car $(p)$ dynamics is defined by the conditional Laplace transforms:

$$
\begin{aligned}
& E_{t}\left[\exp \left(u_{1} x_{1, t+1}\right) \mid x_{2, t+1}, \underline{x_{1, t}}, \underline{x_{2, t}}\right]=\exp \left[a_{1}\left(u_{1}\right)\left(\beta_{o} x_{2, t+1}+\beta_{11}^{\prime} X_{1 t}+\beta_{12}^{\prime} X_{2 t}\right)+b_{1}\left(u_{1}\right)\right], \\
& E_{t}\left[\exp \left(u_{2} x_{2, t+1}\right) \mid \underline{x_{1, t}}, \underline{x_{2, t}}\right]=\exp \left[a_{2}\left(u_{2}\right)\left(\beta_{21}^{\prime} X_{1 t}+\beta_{22}^{\prime} X_{2 t}\right)+b_{2}\left(u_{2}\right)\right],
\end{aligned}
$$

where the $\beta_{i j}$ are $p$-vectors. It is easily seen that the process $\tilde{x_{t}}$ is a $\operatorname{Car}(p)$ process with a conditional Laplace transform given by (23) in which $y_{1, t}$ is replaced by $X_{1 t}$ and $y_{2, t}$ by $X_{2 t}$ and the $\beta_{i j}$ by the $\beta_{i j}^{\prime}$, i.e.

$$
\begin{gathered}
E\left[\exp \left(u^{\prime} \tilde{x}_{t+1}\right) \mid \underline{\left.\tilde{x}_{t}\right]=\exp \{[}\left[a_{1}\left(u_{1}\right) \beta_{11}+a_{2}\left(u_{2}+a_{1}\left(u_{1}\right) \beta_{o}\right) \beta_{21}\right]^{\prime} X_{1 t}\right. \\
+\left[a_{1}\left(u_{1}\right) \beta_{12}+a_{2}\left(u_{2}+a_{1}\left(u_{1}\right) \beta_{o}\right) \beta_{22}\right]^{\prime} X_{2 t} \\
\left.+b_{1}\left(u_{1}\right)+b_{2}\left(u_{2}+a_{1}\left(u_{1}\right) \beta_{o}\right)\right\} .
\end{gathered}
$$

From the properties of $\operatorname{Car}(p)$ processes we get a representation of the form:

$$
\left\{\begin{array}{l}
x_{1, t+1}=\alpha_{1}+\alpha_{o} x_{2, t+1}+\alpha_{11}^{\prime} X_{1 t}+\alpha_{12}^{\prime} X_{2 t}+\varepsilon_{1, t+1} \\
x_{2, t+1}=\alpha_{2}+\alpha_{21}^{\prime} X_{1 t}+\alpha_{22}^{\prime} X_{2 t}+\varepsilon_{2, t+1}
\end{array}\right.
$$


where the errors terms satisfy :

$$
\begin{aligned}
& E\left[\varepsilon_{1, t+1} \mid x_{2, t+1}, \underline{\tilde{x_{t}}}\right]=0 \\
& E\left[\varepsilon_{2, t+1} \mid \underline{\tilde{x_{t}}}\right]=0 ;
\end{aligned}
$$

in particular, we get

$$
\begin{aligned}
E\left[\varepsilon_{1, t+1} \mid \underline{\tilde{x_{t}}}\right] & =0 \\
E\left[\varepsilon_{2, t+1} \mid \underline{\tilde{x_{t}}}\right] & =0 \\
\operatorname{Cov}\left(\varepsilon_{1, t+1}, \varepsilon_{2, t+1}\right) & =E\left(\varepsilon_{1, t+1} \varepsilon_{2, t+1} \mid \underline{\tilde{x_{t}}}\right) \\
& =E\left[\varepsilon_{2, t+1} E\left(\varepsilon_{1, t+1} \mid x_{2, t+1}, \underline{\tilde{x}_{t}}\right) \mid \underline{\tilde{x}_{t}}\right] \\
& =0 .
\end{aligned}
$$

So, the error terms are non correlated, conditionally heteroscedastic, martingale differences. In particular, in the stationary case, $\varepsilon_{1, t}$ and $\varepsilon_{2, t}$ are uncorrelated weak white noises and (26) is a weak recursive $\operatorname{VAR}(p)$ representation of the process $\tilde{x_{t}}$.

In the rest of the paper we will consider two important particular cases.

\section{a) Normal $\operatorname{VAR}(p)$ or $\operatorname{VARN}(p)$ processes}

In this case the conditional distributions defined by (20) are gaussian, with affine expectations and fixed variances. In other words:

$$
\begin{aligned}
& a_{1}\left(u_{1}\right)=\rho_{1} u_{1}, b_{1}\left(u_{1}\right)=\nu_{1} u_{1}+\frac{\sigma_{1}^{2} u_{1}^{2}}{2} \\
& a_{2}\left(u_{2}\right)=\rho_{2} u_{2}, b_{2}\left(u_{2}\right)=\nu_{2} u_{2}+\frac{\sigma_{2}^{2} u_{2}^{2}}{2} .
\end{aligned}
$$

Using the notations $\varphi_{o}=\rho_{1} \beta_{o}, \varphi_{11}=\rho_{1} \beta_{11}, \varphi_{12}=\rho_{1} \beta_{12}, \varphi_{21}=\rho_{2} \beta_{21}, \varphi_{22}=\rho_{2} \beta_{22}$, we have the following strong $\operatorname{VAR}(p)$ recursive representation for the process $\tilde{x}_{t}=\left(x_{1, t}, x_{2, t}\right)^{\prime}$ :

$$
\left\{\begin{array}{l}
x_{1, t+1}=\nu_{1}+\varphi_{o} x_{2, t+1}+\varphi_{11}^{\prime} X_{1 t}+\varphi_{12}^{\prime} X_{2 t}+\sigma_{1} \eta_{1, t+1} \\
x_{2, t+1}=\nu_{2}+\varphi_{21}^{\prime} X_{1 t}+\varphi_{22}^{\prime} X_{2 t}+\sigma_{2} \eta_{2, t+1}
\end{array}\right.
$$

where $\eta_{t}=\left(\eta_{1, t}, \eta_{2, t}\right)^{\prime}$ is a bivariate gaussian white noise distributed as $\mathcal{N}\left(0, I_{2}\right)$, where $I_{2}$ denotes the $(2 \times 2)$ identity matrix.

\section{b) $\operatorname{Gamma} \operatorname{VAR}(p)$ or $\operatorname{VARG}(p)$ processes}

In this case we have:

$$
\begin{aligned}
& a_{1}\left(u_{1}\right)=\frac{\rho_{1} u_{1}}{1-u_{1} \mu_{1}}, b_{1}\left(u_{1}\right)=-\nu_{1} \log \left(1-u_{1} \mu_{1}\right) \\
& a_{2}\left(u_{2}\right)=\frac{\rho_{2} u_{2}}{1-u_{2} \mu_{2}}, b_{2}\left(u_{2}\right)=-\nu_{2} \log \left(1-u_{2} \mu_{2}\right),
\end{aligned}
$$

and the process $\tilde{x_{t}}=\left(x_{1, t}, x_{2, t}\right)^{\prime}$ has the following weak $\operatorname{VAR}(p)$ representation (using the same notation as above, and where all the parameters are positive):

$$
\left\{\begin{array}{l}
x_{1, t+1}=\nu_{1} \mu_{1}+\varphi_{o} x_{2, t+1}+\varphi_{11}^{\prime} X_{1 t}+\varphi_{12}^{\prime} X_{2 t}+\xi_{1, t+1} \\
x_{2, t+1}=\nu_{2} \mu_{2}+\varphi_{21}^{\prime} X_{1 t}+\varphi_{22}^{\prime} X_{2 t}+\xi_{2, t+1}
\end{array}\right.
$$


where $\xi_{1, t}$ and $\xi_{2, t}$ are non correlated, conditionally heteroscedastic, martingale differences. The conditional variances of $\xi_{1, t+1}$ and $\xi_{2, t+1}$ are given by:

$$
\begin{aligned}
& V\left[\xi_{1, t+1} \mid \underline{\tilde{x}_{t}}\right]=\nu_{1} \mu_{1}^{2}+2 \mu_{1}\left[\varphi_{o}\left(\nu_{2} \mu_{2}+\varphi_{21}^{\prime} X_{1 t}+\varphi_{22}^{\prime} X_{2 t}\right)+\varphi_{11}^{\prime} X_{1 t}+\varphi_{12}^{\prime} X_{2 t}\right] \\
& V\left[\xi_{2, t+1} \mid \underline{\tilde{x}_{t}}\right]=\nu_{2} \mu_{2}^{2}+2 \mu_{2}\left(\varphi_{21}^{\prime} X_{1 t}+\varphi_{22}^{\prime} X_{2 t}\right) .
\end{aligned}
$$

It is important to stress that the components of this $\operatorname{VARG}(p)$ process are positive $^{6}$.

\subsection{Switching Multivariate Index-Car processes}

Switching regimes can be introduced in a multivariate Index-Car $(p)$ model using a method extending the one retained in the univariate case. If we assume that the functions $b_{1}\left(u_{1}\right), b_{2}\left(u_{2}\right)$ appearing in definition 3 can be written, respectively, as $\tilde{b_{1}}\left(u_{1}\right)^{\prime} \lambda_{1}$ and $\tilde{b_{2}}\left(u_{2}\right)^{\prime} \lambda_{2}$, and if we replace $\lambda_{1}$ and $\lambda_{2}$, respectively by $\Lambda_{1} Z_{t}$ and $\Lambda_{2} Z_{t}$, we obtain the following conditional Laplace transform for the distribution of $\left(x_{1, t+1}, x_{2, t+1}, z_{t+1}\right)$ given $\left(\underline{x_{1, t}}, \underline{x_{2, t}}, \underline{z_{t}}\right)$ :

$$
\begin{aligned}
& E\left[\exp \left(u_{1} x_{1, t+1}+u_{2} x_{2, t+1}+v^{\prime} z_{t+1}\right) \mid \underline{x_{1, t}}, \underline{x_{2, t}}, \underline{z_{t}}\right] \\
& =\exp \left\{\left[a_{1}\left(u_{1}\right) \beta_{11}+a_{2}\left(u_{2}+a_{1}\left(u_{1}\right) \beta_{o}\right) \beta_{21}\right]^{\prime} X_{1 t}\right. \\
& \quad+\left[a_{1}\left(u_{1}\right) \beta_{12}+a_{2}\left(u_{2}+a_{1}\left(u_{1}\right) \beta_{o}\right) \beta_{22}\right]^{\prime} X_{2 t} \\
& \left.\quad+\left[e_{1}^{\prime} \otimes a_{z}(v, \pi)^{\prime}+\tilde{b_{1}}\left(u_{1}\right)^{\prime} \Lambda_{1}+\tilde{b_{2}}\left(u_{2}+a_{1}\left(u_{1}\right) \beta_{o}\right)^{\prime} \Lambda_{2}\right] Z_{t}\right\},
\end{aligned}
$$

where $a_{z}(v, \pi)$ is given in Proposition 1 . So we obtain a multivariate $\operatorname{Car}(p+1)$ process.

Proposition 4 : The Laplace transform of $\left(x_{1, t+1}, x_{2, t+1}, z_{t+1}\right)$, conditionally to $\left(\underline{x_{1, t}}, \underline{x_{2, t}}, \underline{z_{t}}\right)$, has the form given in (34) and the process $\left(x_{1, t}, x_{2, t}, z_{t}\right)$ is $\operatorname{Car}(p+1)$.

\subsection{Examples of Switching Multivariate Index-Car processes}

\section{a. Gaussian case}

Taking

$$
\begin{aligned}
& a_{1}\left(u_{1}\right)=\rho_{1} u_{1}, b_{1}\left(u_{1}\right)=\nu_{1} u_{1}+\frac{\sigma_{1}^{2}}{2} u_{1}^{2}, \tilde{b_{1}}\left(u_{1}\right)^{\prime}=\left(u_{1}, \frac{u_{1}^{2}}{2}\right), \\
& a_{2}\left(u_{2}\right)=\rho_{2} u_{2}, b_{2}\left(u_{2}\right)=\nu_{2} u_{2}+\frac{\sigma_{2}^{2}}{2} u_{2}^{2}, \tilde{b_{2}}\left(u_{2}\right)^{\prime}=\left(u_{2}, \frac{u_{2}^{2}}{2}\right), \\
& \Lambda_{1}=\left(\begin{array}{c}
\lambda_{11}^{\prime} \\
\lambda_{12}^{\prime}
\end{array}\right), \Lambda_{2}=\left(\begin{array}{c}
\lambda_{21}^{\prime} \\
\lambda_{22}^{\prime}
\end{array}\right),
\end{aligned}
$$

and using the notations $\varphi_{o}=\rho_{1} \beta_{o}, \varphi_{11}=\rho_{1} \beta_{11}, \varphi_{12}=\rho_{1} \beta_{12}, \varphi_{21}=\rho_{2} \beta_{21}, \varphi_{22}=\rho_{2} \beta_{22}$, we obtain the Switching $\operatorname{VARN}(p)$ model:

$$
\left\{\begin{array}{l}
x_{1, t+1}=\lambda_{11}^{\prime} Z_{t}+\varphi_{o} x_{2, t+1}+\varphi_{11}^{\prime} X_{1 t}+\varphi_{12}^{\prime} X_{2 t}+\left(\lambda_{12}^{\prime} Z_{t}\right)^{1 / 2} \eta_{1, t+1} \\
x_{2, t+1}=\lambda_{21}^{\prime} Z_{t}+\varphi_{21}^{\prime} X_{1 t}+\varphi_{22}^{\prime} X_{2 t}+\left(\lambda_{22}^{\prime} Z_{t}\right)^{1 / 2} \eta_{2, t+1}
\end{array}\right.
$$

\footnotetext{
${ }^{6}$ In a recent paper Dai, Le and Singleton (2006) propose a multivariate conditionally Gaussian term structure model where nonlinearities are introduced in the (latent) state-factor (historical and risk-neutral) dynamics by means of stochastic volatility factors; the joint risk-neutral dynamics of these volatility factors is described by a particular VARG(1) process with conditionally independent components $\left[\varphi_{o}=0\right.$ in our system (32) notation].
} 
where $\eta_{t}=\left(\eta_{1, t}, \eta_{2, t}\right)^{\prime}$ is a gaussian white noise distributed as $\mathcal{N}\left(0, I_{2}\right), Z_{t}=\left(z_{t}^{\prime}, \ldots, z_{t-p}^{\prime}\right)^{\prime}$, and where $z_{t}$ is a homogeneous $J$-state Markov chain with transition probability $\pi\left(e_{i}, e_{j}\right)$. Note that (35) can also be written as:

$$
\left\{\begin{array}{l}
x_{1, t+1}=\tilde{\lambda}_{11}^{\prime} Z_{t}+\tilde{\varphi}_{11}^{\prime} X_{1 t}+\tilde{\varphi}_{12}^{\prime} X_{2 t}+\varphi_{o}\left(\lambda_{22}^{\prime} Z_{t}\right)^{1 / 2} \eta_{2, t+1}+\left(\lambda_{12}^{\prime} Z_{t}\right)^{1 / 2} \eta_{1, t+1} \\
x_{2, t+1}=\lambda_{21}^{\prime} Z_{t}+\varphi_{21}^{\prime} X_{1 t}+\varphi_{22}^{\prime} X_{2 t}+\left(\lambda_{22}^{\prime} Z_{t}\right)^{1 / 2} \eta_{2, t+1}
\end{array}\right.
$$

with $\tilde{\lambda}_{11}=\lambda_{11}+\varphi_{o} \lambda_{21}, \tilde{\varphi}_{11}=\varphi_{11}+\varphi_{o} \varphi_{21}, \tilde{\varphi}_{12}=\varphi_{12}+\varphi_{o} \varphi_{22}$ or, with obvious notations

$$
\tilde{x}_{t+1}=\tilde{\lambda}^{\prime} Z_{t}+\tilde{\Phi}^{\prime} \tilde{X}_{t}+\left[\begin{array}{cc}
\left(\lambda_{12}^{\prime} Z_{t}\right)^{1 / 2} & \varphi_{o}\left(\lambda_{22}^{\prime} Z_{t}\right)^{1 / 2} \\
0 & \left(\lambda_{22}^{\prime} Z_{t}\right)^{1 / 2}
\end{array}\right] \eta_{t+1}
$$

\section{b. Gamma case}

If we take

$$
\begin{aligned}
& a_{1}\left(u_{1}\right)=\frac{\rho_{1} u_{1}}{1-u_{1} \mu_{1}}, \quad b_{1}\left(u_{1}\right)=-\nu_{1} \log \left(1-u_{1} \mu_{1}\right), \tilde{b_{1}}\left(u_{1}\right)=\log \left(1-u_{1} \mu_{1}\right), \\
& a_{2}\left(u_{2}\right)=\frac{\rho_{2} u_{2}}{1-u_{2} \mu_{2}}, \quad b_{2}\left(u_{2}\right)=-\nu_{2} \log \left(1-u_{2} \mu_{2}\right), \quad \tilde{b_{2}}\left(u_{2}\right)=\log \left(1-u_{2} \mu_{2}\right),
\end{aligned}
$$

we obtain the positive Switching $\operatorname{VARG}(p)$ model

$$
\left\{\begin{array}{l}
x_{1, t+1}=\mu_{1} \Lambda_{1}^{\prime} Z_{t}+\varphi_{o} x_{2, t+1}+\varphi_{11}^{\prime} X_{1 t}+\varphi_{12}^{\prime} X_{2 t}+\xi_{1, t+1} \\
x_{2, t+1}=\mu_{2} \Lambda_{2}^{\prime} Z_{t}+\varphi_{21}^{\prime} X_{1 t}+\varphi_{22}^{\prime} X_{2 t}+\xi_{2, t+1}
\end{array}\right.
$$

where $\xi_{1, t}$ and $\xi_{2, t}$ are non correlated, conditionally heteroscedastic, martingale differences, the conditional variances being respectively given by:

$$
\begin{aligned}
& V\left[\xi_{1, t+1} \mid \underline{\tilde{x}_{t}}\right]=\Lambda_{1}^{\prime} Z_{t} \mu_{1}^{2}+2 \mu_{1}\left[\varphi_{o}\left(\Lambda_{2}^{\prime} Z_{t} \mu_{2}+\varphi_{21}^{\prime} X_{1 t}+\varphi_{22}^{\prime} X_{2 t}\right)+\varphi_{11}^{\prime} X_{1 t}+\varphi_{12}^{\prime} X_{2 t}\right] \\
& V\left[\xi_{2, t+1} \mid \underline{\tilde{x}_{t}}\right]=\Lambda_{2}^{\prime} Z_{t} \mu_{2}^{2}+2 \mu_{2}\left(\varphi_{21}^{\prime} X_{1 t}+\varphi_{22}^{\prime} X_{2 t}\right) .
\end{aligned}
$$

\section{Switching Autoregressive Normal (SARN) Factor-Based Term Structure Model of order $p$}

We first consider the case of univariate latent factor; the observable factor case and the multivariate case will be discussed, respectively, in Sections 3.7 and 3.8.

\subsection{The historical dynamics}

The first set of assumptions of a $\operatorname{SARN}(p)$ Term Structure model deals with the historical dynamics. We assume that the historical dynamics of the latent factor $x_{t}$ is given by

$$
x_{t+1}=\nu\left(Z_{t}\right)+\varphi_{1}\left(Z_{t}\right) x_{t}+\ldots+\varphi_{p}\left(Z_{t}\right) x_{t+1-p}+\sigma\left(Z_{t}\right) \varepsilon_{t+1},
$$

where $\varepsilon_{t+1}$ is a gaussian white noise with $\mathcal{N}(0,1)$ distribution, $Z_{t}=\left(z_{t}^{\prime}, \ldots, z_{t-p}^{\prime}\right)^{\prime}$, and $z_{t}$ is a $J$-state non-homogeneous Markov chain such that $P\left(z_{t+1}=e_{j} \mid z_{t}=e_{i} ; \underline{x_{t}}\right)=\pi\left(e_{i}, e_{j} ; X_{t}\right)\left(e_{i}\right.$ is 
the $i^{t h}$ column of the identity matrix $\left.I_{J}\right)$ and $X_{t}=\left(x_{t}, \ldots, x_{t+1-p}\right)^{\prime}$. Equation (40) will be also written

$$
x_{t+1}=\nu\left(Z_{t}\right)+\varphi\left(Z_{t}\right)^{\prime} X_{t}+\sigma\left(Z_{t}\right) \varepsilon_{t+1},
$$

where $\varphi\left(Z_{t}\right)=\left(\varphi_{1}\left(Z_{t}\right), \ldots, \varphi_{p}\left(Z_{t}\right)\right)^{\prime}$. This model can also be rewritten in the following vectorial form:

$$
X_{t+1}=\Phi\left(Z_{t}\right) X_{t}+\left[\nu\left(Z_{t}\right)+\sigma\left(Z_{t}\right) \varepsilon_{t+1}\right] e_{1}
$$

where

$$
\Phi\left(Z_{t}\right)=\left[\begin{array}{cccc}
\varphi_{1}\left(Z_{t}\right) & \ldots & \ldots & \varphi_{p}\left(Z_{t}\right) \\
1 & 0 & \ldots & 0 \\
0 & 1 & \ldots & 0 \\
\vdots & & \ddots & \vdots \\
0 & \ldots & 1 & 0
\end{array}\right]
$$

is a $(p \times p)$-matrix, and where $e_{1}$ is the first column of the identity matrix $I_{p}$. Note that, since the coefficients $\varphi_{i}$ are allowed to depend on $Z_{t}$ and since the Markov chain $z_{t}$ may not be homogeneous, the dynamics of $\left(x_{t}, z_{t}\right)$ is not Car in general.

\subsection{The Stochastic Discount Factor}

The second element of a $\operatorname{SARN}(p)$ modeling is the SDF. We denote by $M_{t, t+1}$ the stochastic discount factor (SDF) between the date $t$ and $t+1$ and in order to get time-varying risk-premia we specify it as an exponential affine function of the variables $\left(x_{t+1}, z_{t+1}\right)$ but with coefficients depending on the information at time $t$. More precisely we assume that:

$$
M_{t, t+1}=\exp \left[-c^{\prime} X_{t}-d^{\prime} Z_{t}+\Gamma\left(Z_{t}, X_{t}\right) \varepsilon_{t+1}-\frac{1}{2} \Gamma\left(Z_{t}, X_{t}\right)^{2}-\delta\left(Z_{t}, X_{t}\right)^{\prime} z_{t+1}\right]
$$

where $\Gamma\left(Z_{t}, X_{t}\right)=\gamma\left(Z_{t}\right)+\tilde{\gamma}^{\prime}\left(Z_{t}\right) X_{t}$ and $\delta\left(Z_{t}, X_{t}\right)=\left[\delta_{1}\left(Z_{t}, X_{t}\right), \ldots, \delta_{J}\left(Z_{t}, X_{t}\right)\right]^{\prime}$. Our specification of the factor risk correction coefficient $\Gamma\left(Z_{t}, X_{t}\right)$ extends to the multi-lag case the regime-switching essentially affine specification proposed by Dai, Singleton and Yang (2006). Bansal and Zhou (2002) assume a market price of factor risk proportional to factor volatilities (completely affine specification $)^{7}$. Duffee (2002) and Dai and Singleton (2002) show that, among single-regime continuous time term structure models, essentially affine specifications for the market price of factor risk explain dynamic properties of yield curves better than the completely affine specifications of multifactor CIR models. In Sections 6.6 and 6.7 we will find confirmation of this result and, in Section 6.8, we will see how pricing the risk associated to the second lagged factor value is crucial in explaining the long horizon Expectation Hypothesis Puzzle. Naik and Lee (1997), Bansal and Zhou (2002) and Ang and Bekaert (2005) consider the $j^{\text {th }}$-regime risk correction coefficient $\delta_{j}\left(Z_{t}, X_{t}\right)=0$ for each $j \in\{1, \ldots, J\}$, while, pricing regime-shift risk, gives to our approach the possibility to better fit interest rates dynamics [see Section 6.8].

It is well known that the existence of a positive stochastic discount factor is equivalent to the absence of arbitrage opportunity condition and that the price $p_{t}$ at $t$ of a payoff $W_{t+1}$ at $t+1$ is given by:

$$
p_{t}=E\left[M_{t, t+1} W_{t+1} \mid I_{t}\right]=E_{t}\left[M_{t, t+1} W_{t+1}\right],
$$

\footnotetext{
${ }^{7} \mathrm{~A}$ market price of factor risk is said to be essentially affine when it is proportional to both factor volatilities and state-factors [see Duffee (2002), Dai and Singleton (2003)].
} 
where the information $I_{t}$, available for the investors at the date $t$, is given by $\left(\underline{x_{t}}, \underline{z_{t}}\right)$. More generally, the price $p_{t, h}$ at $t$ of an asset paying $W_{t+h}$ at $t+h$ is:

$$
p_{t, h}=E_{t}\left[M_{t, t+1} \cdot \ldots \cdot M_{t+h-1, t+h} W_{t+h}\right] .
$$

Using the absence of arbitrage assumption for the short-term interest rate between $t$ and $t+1$, denoted by $r_{t+1}$ and known at $t$, we get:

$$
\begin{aligned}
\exp \left(-r_{t+1}\right) & =E_{t}\left(M_{t, t+1}\right) \\
& =\exp \left[-c^{\prime} X_{t}-d^{\prime} Z_{t}\right] \times \sum_{j=1}^{J} \pi\left(e_{i}, e_{j} ; X_{t}\right) \exp \left[-\delta\left(Z_{t}, X_{t}\right)^{\prime} e_{j}\right],
\end{aligned}
$$

and assuming the normalization condition:

$$
\sum_{j=1}^{J} \pi\left(e_{i}, e_{j} ; X_{t}\right) \exp \left[-\delta\left(Z_{t}, X_{t}\right)^{\prime} e_{j}\right]=1 \quad \forall Z_{t}, X_{t}
$$

we obtain:

$$
r_{t+1}=c^{\prime} X_{t}+d^{\prime} Z_{t}
$$

\subsection{Risk premia}

In this paper we will use the following definition of a risk premium.

Definition 4 : Let $p_{t}$ the price of a given asset at time $t$. The risk premium of this asset between $t$ and $t+1$ is $\omega_{t}=\log \left(E_{t} p_{t+1}\right)-\log p_{t}-r_{t+1}$.

Using this definition we obtain interpretations of the $\Gamma$ and $\delta$ functions appearing in the SDF which generalize that obtained by Dai, Singleton and Yang (2006).

Proposition 5 : The risk premium between $t$ and $t+1$ of an asset providing the payoff $\exp \left(-\theta x_{t+1}\right)$ at $t+1$ is :

$$
\omega_{t}(\theta)=\theta \Gamma\left(Z_{t}, X_{t}\right) \sigma\left(Z_{t}\right) .
$$

Therefore, $\theta, \Gamma\left(Z_{t}, X_{t}\right)$ and $\sigma\left(Z_{t}\right)$ can be seen respectively as a risk sensitivity of the asset, a risk price and a risk measure. [Proof : see Appendix 2.]

Proposition 6 : If we consider a digital asset providing one money unit at $t+1$ if $z_{t+1}=e_{j}$, its risk premium between $t$ and $t+1$ is given by :

$$
\omega_{t}(\theta)=\delta_{j}\left(Z_{t}, X_{t}\right)
$$

and the $j^{\text {th }}$ component of $\delta$ can be seen as the risk premium associated with the digital asset [Proof: see Appendix 2].

We observe that, in general, the magnitude of the risk premium $\omega_{t}(\theta)$ is not just depending on the currently observed values $x_{t}$ and $z_{t}$, but it includes the present and past values of both factors, that is, it is a function of the larger information represented by $X_{t}$ and $Z_{t}$.

\subsection{Risk-Neutral dynamics}

The assumptions on the historical dynamics and on the SDF imply a risk-neutral dynamics. The probability density function of the one-period conditional risk-neutral probability with respect to the corresponding historical probability is $\frac{M_{t, t+1}}{E_{t}\left(M_{t, t+1}\right)}=\exp \left(r_{t+1}\right) M_{t, t+1}$. Note that using $E_{t}^{\mathbb{Q}}$ as the 
conditional expectation with respect to this risk-neutral distribution, the risk-premium $\omega_{t}$ can be written $\log \left(E_{t} p_{t+1}\right)-\log \left(E_{t}^{\mathbb{Q}} p_{t+1}\right)$.

Proposition 7 : The risk-neutral dynamics of the process $\left(x_{t}, z_{t}\right)$ is given by:

$$
x_{t+1}=\nu\left(Z_{t}\right)+\gamma\left(Z_{t}\right) \sigma\left(Z_{t}\right)+\left[\varphi\left(Z_{t}\right)+\tilde{\gamma}\left(Z_{t}\right) \sigma\left(Z_{t}\right)\right]^{\prime} X_{t}+\sigma\left(Z_{t}\right) \xi_{t+1},
$$

where $\xi_{t+1}$ is (under $\left.\mathbb{Q}\right)$ a gaussian white noise with $\mathcal{N}(0,1)$ distribution, and where $Z_{t}=\left(z_{t}^{\prime}, \ldots, z_{t-p}^{\prime}\right)^{\prime}$, $z_{t}$ being a Markov chain such that:

$$
\mathbb{Q}\left(z_{t+1}=e_{j} \mid \underline{z_{t}} ; \underline{x_{t}}\right)=\pi\left(z_{t}, e_{j} ; X_{t}\right) \exp \left[\left(-\delta\left(Z_{t}, X_{t}\right)\right)^{\prime} e_{j}\right] .
$$

Note that, from (44), these probabilities add to one [Proof : see Appendix 3].

In order to get a generalized linear term structure we impose that the risk-neutral dynamics is switching regime gaussian $\operatorname{Car}(p)$. Using (13), this impose that the dynamics has to satisfy the following specification:

$$
x_{t+1}=\nu^{*^{\prime}} Z_{t}+\varphi^{*^{\prime}} X_{t}+\left(\sigma^{*^{\prime}} Z_{t}\right) \xi_{t+1},
$$

where $Z_{t}=\left(z_{t}^{\prime}, \ldots, z_{t-p}^{\prime}\right)^{\prime}$, with $z_{t}$ a $J$-state Markov chain such that ${ }^{8}$

$$
\mathbb{Q}\left(z_{t+1}=e_{j} \mid z_{t}=e_{i}\right)=\pi^{*}\left(e_{i}, e_{j}\right) .
$$

From Proposition 7, this implies the following restrictions on the historical dynamics and on the SDF:

i) $\sigma\left(Z_{t}\right)=\sigma^{*^{\prime}} Z_{t}$ : the historical stochastic volatility must be linear in $Z_{t}$;

ii)

$$
\gamma\left(Z_{t}\right)=\frac{\nu^{*^{\prime}} Z_{t}-\nu\left(Z_{t}\right)}{\sigma^{*^{\prime}} Z_{t}}:
$$

for a given historical stochastic drift $\nu\left(Z_{t}\right)$ and stochastic volatility $\sigma^{*^{\prime}} Z_{t}$, the coefficient $\gamma\left(Z_{t}\right)$ belongs to the previous family indexed by the free parameter vector $\nu^{*}$.

iii)

$$
\tilde{\gamma}\left(Z_{t}\right)=\frac{\varphi^{*}-\varphi\left(Z_{t}\right)}{\sigma^{*^{\prime}} Z_{t}}:
$$

for a given historical stochastic slope parameter $\varphi\left(Z_{t}\right)$ and stochastic volatility $\sigma^{*^{\prime}} Z_{t}$ the coefficient vector $\tilde{\gamma}\left(Z_{t}\right)$ belongs to the previous family indexed by the free parameter vector $\varphi^{*}$.

iv)

$$
\delta_{j}\left(Z_{t}, X_{t}\right)=\log \left[\frac{\pi\left(z_{t}, e_{j} ; X_{t}\right)}{\pi^{*}\left(z_{t}, e_{j}\right)}\right]:
$$

for a given historical transition matrix $\pi\left(z_{t}, e_{j} ; X_{t}\right)$, the coefficient $\delta_{j}\left(Z_{t}, X_{t}\right)$ depends on $z_{t}$ only and belongs to the previous family indexed by the entries $\pi^{*}\left(z_{t}, e_{j}\right)$ of a transition matrix.

\footnotetext{
${ }^{8}$ Ang and Bekaert (2005) also assume their counterpart to $\varphi^{*}$ to be constant over time, and a homogeneous (risk-neutral) transition matrix for $\left(z_{t}\right)$. Bansal and Zhou (2002) consider $\mathbb{Q}\left(z_{t+1}=e_{j} \mid z_{t}=e_{i}\right)=\pi^{*}\left(e_{i}, e_{j}\right)$, but they allow the risk-neutral autoregressive matrix to switch over time $\left[\varphi^{*}\left(z_{t+1}\right)\right.$, in our notation] : this feature leads their approach to use a log-linear approximation in order to find an explicit (approximate) pricing formula. This kind of log-linear approximation, in a general equilibrium square-root term structure model, is also used by Wu and Zeng (2005).
} 
Note that condition $i v$ ) implies that the risk premia coefficients $\delta_{j}, j \in\{1, \ldots, J\}$, cannot be all positive [or all negative] since this would imply $\pi\left(z_{t}, e_{j} ; X_{t}\right)>\pi^{*}\left(z_{t}, e_{j}\right), \forall j$ [or $\pi\left(z_{t}, e_{j} ; X_{t}\right)<$ $\left.\pi^{*}\left(z_{t}, e_{j}\right), \forall j\right]$, which is impossible since $\sum_{j=1}^{J} \pi\left(z_{t}, e_{j} ; X_{t}\right)=\sum_{j=1}^{J} \pi^{*}\left(z_{t}, e_{j}\right)=1$. Also note that condition $i v$ ) implies the normalization condition (44).

\subsection{The Generalised Linear Term Structure}

We have seen in the previous section that the risk-neutral dynamics is defined by relations (49), (50); relation (49) can be rewritten:

$$
X_{t+1}=\Phi^{*} X_{t}+\left[\nu^{*^{\prime}} Z_{t}+\left(\sigma^{*^{\prime}} Z_{t}\right) \xi_{t+1}\right] e_{1}
$$

where

$$
\begin{gathered}
\Phi^{*}=\left[\begin{array}{cccc}
\varphi_{1}^{*} & \ldots & \ldots & \varphi_{p}^{*} \\
1 & 0 & \ldots & 0 \\
0 & 1 & \ldots & 0 \\
\vdots & & \ddots & \vdots \\
0 & \ldots & 1 & 0
\end{array}\right] \text { is a }(p \times p)-\text { matrix } \\
X_{t}=\left(x_{t}, \ldots, x_{t+1-p}\right)^{\prime}
\end{gathered}
$$

and where $e_{1}$ is the first column of the identity matrix $I_{p}$.

Denoting by $B(t, h)$ the price at $t$ of a zero-coupon with residual maturity $h$, we have the following result.

Proposition 8 : In the univariate $\operatorname{SARN}(p)$ Factor-Based Term Structure Model the price at date $t$ of the zero-coupon bond with residual maturity $h$ is :

$$
B(t, h)=\exp \left(C_{h}^{\prime} X_{t}+D_{h}^{\prime} Z_{t}\right), \text { for } h \geq 1,
$$

where the vectors $C_{h}$ and $D_{h}$ satisfy the following recursive equations :

$$
\left\{\begin{array}{l}
C_{h}=\Phi^{*^{\prime}} C_{h-1}-c \\
D_{h}=-d+C_{1, h-1} \nu^{*}+\frac{1}{2} C_{1, h-1}^{2} \sigma^{* 2}+\tilde{D}_{h-1}+F\left(D_{1, h-1}\right),
\end{array}\right.
$$

where $C_{1, h-1}$ denotes the first component of the $p$-dimensional vector $C_{h-1}, D_{1, h-1}$ and $D_{2, h-1}$ are, respectively, the first $J$-dimensional component and the remaining $(p J)$-dimensional component of $D_{h-1}$, i.e. $D_{h-1}=\left(D_{1, h-1}^{\prime}, D_{2, h-1}^{\prime}\right)^{\prime}, \tilde{D}_{h-1}=\left(D_{2, h-1}^{\prime}, 0\right)^{\prime}$, and where $F\left(D_{1, h-1}\right)=e_{1} \otimes a_{z}\left(D_{1, h-1}\right.$, $\left.\pi^{*}\right), e_{1}$ being the vector $(1,0, \ldots, 0)^{\prime}$ of size $(p+1)$ and $a_{z}$ is the $J$-vector given in Proposition 1 ; $\sigma^{* 2}$ is the vector whose components are the squares of the entries of $\sigma^{*}$. The initial conditions are $C_{0}=0, D_{0}=0$ (or $C_{1}=-c, D_{1}=-d$ ) [Proof : see Appendix 4].

For clarity we give again the expression of $a_{z}\left(D_{1, h-1}, \pi^{*}\right)$ :

$$
\begin{aligned}
& a_{z}\left(D_{1, h-1}, \pi^{*}\right) \\
= & {\left[\log \left(\sum_{j=1}^{J} \exp \left(D_{1, h-1}^{\prime} e_{j}\right) \pi^{*}\left(e_{1}, e_{j}\right)\right), \ldots, \log \left(\sum_{j=1}^{J} \exp \left(D_{1, h-1}^{\prime} e_{j}\right) \pi^{*}\left(e_{J}, e_{j}\right)\right)\right]^{\prime} . }
\end{aligned}
$$


From Proposition 8 we see that the yields to maturity are:

$$
\begin{aligned}
R(t, h) & =-\frac{1}{h} \log B(t, h) \\
& =-\frac{C_{h}^{\prime}}{h} X_{t}-\frac{D_{h}^{\prime}}{h} Z_{t}, \quad h \geq 1 .
\end{aligned}
$$

So, they are linear functions of the $p$-dimensional vector $X_{t}$ and of the $(p+1) J$-dimensional vector $Z_{t}$. This means that, the term structure at date $t$ depends on the present and past values of $x_{t}$ and $z_{t}$, and not just on their values in $t$. Moreover, we observe that there is, in general, instantaneous causality between $x_{t}$ and $z_{t}$.

\subsection{The Switching VARMA yield curve process}

The result presented in Proposition 8 describes, conditionally to $X_{t}$ and $Z_{t}$, the yields as a deterministic function of the time to maturity $h$, for a fixed date $t$. Nevertheless, in many financial and economic contexts one needs to study the effects, of a shock in the state variables, on the yield curve at different future times and for several maturities (e.g.: a Central Bank that needs to set a monetary policy). This means that we are interested in the dynamics of the process $R_{\mathcal{H}}=[R(t, h), 0 \leq t<T, h \in \mathcal{H}]$, for a given set of residual time to maturities $\mathcal{H}=(1, \ldots, H)$.

If we consider a fixed $h$, the process $R=[R(t, h), 0 \leq t<T]$ can be described by the following proposition.

Proposition 9 : For a fixed time to maturity $h$, the process $R=[R(t, h), 0 \leq t<T]$ is, under the historical probability, a Switching $\operatorname{ARMA}(p, p-1)$ process of the following type :

$$
\Psi\left(L, Z_{t}\right) R(t+1, h)=D_{h}(L) \Psi\left(L, Z_{t}\right) z_{t+1}+C_{h}(L) \nu\left(Z_{t}\right)+C_{h}(L)\left[\left(\sigma^{*^{\prime}} Z_{t}\right) \varepsilon_{t+1}\right] .
$$

where

$$
\begin{aligned}
C_{h}(L) & =-\frac{1}{h}\left(C_{1, h}+C_{2, h} L+\ldots+C_{p, h} L^{p-1}\right) \\
D_{h}(L) & =-\frac{1}{h}\left(D_{1, h}+D_{2, h} L+\ldots+D_{p+1, h} L^{p}\right) \\
\Psi\left(L, Z_{t}\right) & =1-\varphi_{1}\left(Z_{t}\right) L-\ldots-\varphi_{p}\left(Z_{t}\right) L^{p},
\end{aligned}
$$

are lag polynomials in the lag operator $L$, and where the AR polynomial $\Psi\left(L, Z_{t}\right)$ applies to $t$ [Proof : see Appendix 5].

Proposition 10 : For a given set of residual time to maturities $\mathcal{H}=(1, \ldots, H)$, the stochastic evolution of the yield curve process $R_{\mathcal{H}}=[R(t, h), 0 \leq t<T, h \in \mathcal{H}]$ takes the following particular Switching $H$-variate $\operatorname{VARMA}(p, p-1)$ representation:

$$
\Psi\left(L, Z_{t}\right)\left(\begin{array}{c}
R(t+1,1) \\
R(t+1,2) \\
\vdots \\
R(t+1, H)
\end{array}\right)=\left(\begin{array}{c}
C_{1}(L) \\
C_{2}(L) \\
\vdots \\
C_{H}(L)
\end{array}\right)\left(\sigma^{*^{\prime}} Z_{t}\right) \varepsilon_{t+1}+\left(\begin{array}{c}
D_{1}(L) \\
D_{2}(L) \\
\vdots \\
D_{H}(L)
\end{array}\right) \Psi\left(L, Z_{t}\right) z_{t+1}+\left(\begin{array}{c}
C_{1}(L) \\
C_{2}(L) \\
\vdots \\
C_{H}(L)
\end{array}\right) \nu\left(Z_{t}\right) .
$$

Similar results are easily obtained in the risk-neutral world. 


\subsection{Endogenous case}

In the previous sections the factor $x_{t}$ was latent. It is often assumed, in term structure models, that the factor $x_{t}$ is the short rate process $r_{t+1}$. In this case the previous results remain valid, the only modification comes from the absence of arbitrage opportunity condition for $r_{t+1}$, which imposes:

$$
c=e_{1}, d=0,
$$

with $e_{1}$ the first column of the identity matrix $I_{p}$; consequently, the initial conditions in the recursive equations of Proposition 8 become:

$$
C_{1}=-e_{1}, D_{1}=0
$$

Moreover, the Switching $\operatorname{ARMA}(p, p-1)$ representation (55), or its analogous in the risk-neutral world, could be used to analyse how a shock on $\varepsilon_{t}$, i.e. on $r_{t+1}=R(t, 1)$, is propagated on the surface $[R(t+\tau, h), \tau \in \mathcal{T}, h \in \mathcal{H}]$, where $\mathcal{T}=\{0, \ldots, T-t-1\}$ and $\mathcal{H}=(1, \ldots, H)$ (for instance when the process $z_{t}$ is latent).

\subsection{Multi-Factor generalization : the $\operatorname{SVARN}(p)$ Factor-Based Term Structure Model}

For sake of notational simplicity we consider the two factor case but an extension to more that two factors is straightforward. The historical dynamics of $\tilde{x}_{t}=\left(x_{1, t}, x_{2, t}\right)^{\prime}$ is a bivariate $\operatorname{SVARN}(p)$ model given by:

$$
\left\{\begin{array}{l}
x_{1, t+1}=\nu_{1}\left(Z_{t}\right)+\varphi_{o}\left(Z_{t}\right) x_{2, t+1}+\varphi_{11}\left(Z_{t}\right)^{\prime} X_{1 t}+\varphi_{12}\left(Z_{t}\right)^{\prime} X_{2 t}+\sigma_{1}\left(Z_{t}\right) \varepsilon_{1, t+1} \\
x_{2, t+1}=\nu_{2}\left(Z_{t}\right)+\varphi_{21}\left(Z_{t}\right)^{\prime} X_{1 t}+\varphi_{22}\left(Z_{t}\right)^{\prime} X_{2 t}+\sigma_{2}\left(Z_{t}\right) \varepsilon_{2, t+1}
\end{array}\right.
$$

where $\varepsilon_{1, t}$ and $\varepsilon_{2, t}$ are independent standard normal white noises, $X_{1 t}=\left(x_{1, t}, \ldots, x_{1, t+1-p}\right)^{\prime}$, $X_{2 t}=\left(x_{2, t}, \ldots, x_{2, t+1-p}\right)^{\prime}, Z_{t}=\left(z_{t}^{\prime}, \ldots, z_{t-p}^{\prime}\right)^{\prime}$, with $z_{t}$ a $J$-state non-homogeneous Markov chain such that $P\left(z_{t+1}=e_{j} \mid z_{t}=e_{i} ; \underline{\tilde{x}_{t}}\right)=\pi\left(e_{i}, e_{j} ; \tilde{X}_{t}\right)$, and where $\tilde{X}_{t}=\left(X_{1 t}^{\prime}, X_{2 t}^{\prime}\right)^{\prime}$. The recursive form (59) is equivalent to the canonical form :

$$
\left\{\begin{array}{l}
x_{1, t+1}=\tilde{\nu}_{1}\left(Z_{t}\right)+\tilde{\varphi}_{11}\left(Z_{t}\right)^{\prime} X_{1 t}+\tilde{\varphi}_{12}\left(Z_{t}\right)^{\prime} X_{2 t}+\sigma_{1}\left(Z_{t}\right) \varepsilon_{1, t+1}+\varphi_{o}\left(Z_{t}\right) \sigma_{2}\left(Z_{t}\right) \varepsilon_{2, t+1} \\
x_{2, t+1}=\nu_{2}\left(Z_{t}\right)+\varphi_{21}\left(Z_{t}\right)^{\prime} X_{1 t}+\varphi_{22}\left(Z_{t}\right)^{\prime} X_{2 t}+\sigma_{2}\left(Z_{t}\right) \varepsilon_{2, t+1},
\end{array}\right.
$$

where $\tilde{\nu}_{1}=\nu_{1}+\varphi_{o} \nu_{2}, \tilde{\varphi}_{11}=\varphi_{11}+\varphi_{o} \varphi_{21}, \tilde{\varphi}_{12}=\varphi_{12}+\varphi_{o} \varphi_{22}$ or, with obvious notations:

$$
\tilde{x}_{t+1}=\tilde{\nu}\left(Z_{t}\right)+\tilde{\Phi}\left(Z_{t}\right) \tilde{X}_{t}+S\left(Z_{t}\right) \varepsilon_{t+1},
$$

where

$$
S\left(Z_{t}\right)=\left[\begin{array}{cc}
\sigma_{1}\left(Z_{t}\right) & \varphi_{o}\left(Z_{t}\right) \sigma_{2}\left(Z_{t}\right) \\
0 & \sigma_{2}\left(Z_{t}\right)
\end{array}\right]
$$

Using the notation

$$
\Gamma\left(Z_{t}, \tilde{X}_{t}\right)=\left[\Gamma_{1}\left(Z_{t}, \tilde{X}_{t}\right), \Gamma_{2}\left(Z_{t}, \tilde{X}_{t}\right)\right]^{\prime}
$$


where $\Gamma_{i}\left(Z_{t}, \tilde{X}_{t}\right)=\gamma_{i}\left(Z_{t}\right)+\tilde{\gamma}_{i}\left(Z_{t}\right)^{\prime} \tilde{X}_{t}, i \in\{1,2\}$ and $\Gamma\left(Z_{t}, \tilde{X}_{t}\right)=\gamma\left(Z_{t}\right)+\tilde{\Gamma}\left(Z_{t}\right) \tilde{X}_{t}$, with $\gamma\left(Z_{t}\right)=$ $\left[\gamma_{1}\left(Z_{t}\right), \gamma_{2}\left(Z_{t}\right)\right]^{\prime}, \tilde{\Gamma}\left(Z_{t}\right)=\left[\tilde{\gamma}_{1}\left(Z_{t}\right)^{\prime}, \tilde{\gamma_{2}}\left(Z_{t}\right)^{\prime}\right]^{\prime}$, the SDF is defined as :

$$
M_{t, t+1}=\exp \left[-c^{\prime} \tilde{X}_{t}-d^{\prime} Z_{t}+\Gamma\left(Z_{t}, \tilde{X}_{t}\right)^{\prime} \varepsilon_{t+1}-\frac{1}{2} \Gamma\left(Z_{t}, \tilde{X}_{t}\right)^{\prime} \Gamma\left(Z_{t}, \tilde{X}_{t}\right)-\delta\left(Z_{t}, \tilde{X}_{t}\right)^{\prime} z_{t+1}\right] .
$$

Assuming the normalization condition (44) and the absence of arbitrage opportunity for $r_{t+1}$ we get:

$$
r_{t+1}=c^{\prime} \tilde{X}_{t}+d^{\prime} Z_{t}
$$

It is also easily seen that the risk premium for an asset providing the payoff $\exp \left(-\theta^{\prime} \tilde{x}_{t+1}\right)$ at $t+1$ is $\omega(\theta)=\theta^{\prime} S\left(Z_{t}\right) \Gamma\left(Z_{t}, \tilde{X}_{t}\right)$ and that the risk premium associated with the digital payoff $\mathbb{I}_{\left(e_{j}\right)}\left(z_{t+1}\right)$ is unchanged.

Proposition 11 : The risk-neutral dynamics of the process $\left(\tilde{x}_{t}, z_{t}\right)$ is given by:

$$
\tilde{x}_{t+1}=\tilde{\nu}\left(Z_{t}\right)+S\left(Z_{t}\right) \gamma\left(Z_{t}\right)+\left[\tilde{\Phi}\left(Z_{t}\right)+S\left(Z_{t}\right) \tilde{\Gamma}\left(Z_{t}, \tilde{X}_{t}\right)\right] \tilde{X}_{t}+S\left(Z_{t}\right) \xi_{t+1}
$$

where $\xi_{t+1}$ is (under $\mathbb{Q}$ ) a bivariate gaussian white noise with $\mathcal{N}\left(0, I_{2}\right)$ distribution, and where $Z_{t}=\left(z_{t}^{\prime}, \ldots, z_{t-p}^{\prime}\right)^{\prime}, z_{t}$ being a Markov chain such that:

$$
\mathbb{Q}\left(z_{t+1}=e_{j} \mid \underline{z_{t}} ; \underline{\tilde{x}_{t}}\right)=\pi\left(z_{t}, e_{j} ; \tilde{X}_{t}\right) \exp \left[\left(-\delta\left(Z_{t}, \tilde{X}_{t}\right)\right)^{\prime} e_{j}\right]
$$

[Proof : see Appendix 6.]

If we want to obtain a Switching bivariate Car process in the risk-neutral world, we must have using (37) :

i)

$$
\begin{aligned}
& \sigma_{1}\left(Z_{t}\right)=\sigma_{1}^{*^{\prime}} Z_{t} \\
& \sigma_{2}\left(Z_{t}\right)=\sigma_{2}^{*^{\prime}} Z_{t} \\
& \varphi_{o}\left(Z_{t}\right)=\varphi_{o}^{*},
\end{aligned}
$$

and, therefore,

$$
S\left(Z_{t}\right)=\left[\begin{array}{cc}
\sigma_{1}^{*^{\prime}} Z_{t} & \varphi_{o}^{*} \sigma_{2}^{*^{\prime}} Z_{t} \\
0 & \sigma_{2}^{*^{\prime}} Z_{t}
\end{array}\right]
$$

ii)

$$
\gamma\left(Z_{t}\right)=\left[S\left(Z_{t}\right)\right]^{-1}\left[\nu^{*} Z_{t}-\tilde{\nu}\left(Z_{t}\right)\right],
$$

where $\nu^{*}$ is a $(2 \times(p+1) J)$-matrix.

iii)

$$
\tilde{\Gamma}\left(Z_{t}\right)=\left[S\left(Z_{t}\right)\right]^{-1}\left[\Phi^{*}-\tilde{\Phi}\left(Z_{t}\right)\right],
$$

where $\Phi^{*}$ is a $(2 \times 2 p)$-matrix.

iv)

$$
\delta_{j}\left(Z_{t}, \tilde{X}_{t}\right)=\log \left[\frac{\pi\left(z_{t}, e_{j} ; \tilde{X}_{t}\right)}{\pi^{*}\left(z_{t}, e_{j}\right)}\right]
$$


The risk-neutral dynamics can be written:

$$
\left\{\begin{array}{l}
x_{1, t+1}=\nu_{1}^{*} Z_{t}+\Phi_{1}^{*} \tilde{X}_{t}+S_{1}^{*}\left(Z_{t}\right) \xi_{t+1} \\
x_{2, t+1}=\nu_{2}^{*} Z_{t}+\Phi_{2}^{*} \tilde{X}_{t}+S_{2}^{*}\left(Z_{t}\right) \xi_{t+1},
\end{array}\right.
$$

where $\nu_{i}^{*}, \Phi_{i}^{*}, S_{i}^{*}$ are the $i^{\text {th }}$ row of $\nu^{*}, \Phi^{*}, S^{*}$, with $i \in\{1,2\}$, or

$$
\tilde{X}_{t+1} \stackrel{\mathbb{Q}}{=} \tilde{\Phi}^{*} \tilde{X}_{t}+\left[\nu_{1}^{*} Z_{t}+S_{1}^{*}\left(Z_{t}\right) \xi_{t+1}\right] e_{1}+\left[\nu_{2}^{*} Z_{t}+S_{2}^{*}\left(Z_{t}\right) \xi_{t+1}\right] e_{p+1},
$$

where $e_{1}$ (respectively, $e_{p+1}$ ) is of size $2 p$, with entries equal to zero except the first (respectively, the $\left.(p+1)^{t h}\right)$ one which is equal to one, and

$$
\tilde{\Phi}^{*}=\left[\begin{array}{cc}
\Phi_{11}^{*} & \Phi_{12}^{*} \\
\tilde{I} & \tilde{\mathbf{0}} \\
\Phi_{21}^{*} & \Phi_{22}^{*} \\
\tilde{\mathbf{0}} & \tilde{I}
\end{array}\right]
$$

where $\Phi_{1}^{*}=\left(\Phi_{11}^{*}, \Phi_{12}^{*}\right), \Phi_{2}^{*}=\left(\Phi_{21}^{*}, \Phi_{22}^{*}\right)$, and where $\tilde{\mathbf{0}}$ is a $[(p-1) \times p]$-matrix of zeros and $\tilde{I}$ is a $[(p-1) \times p]$-matrix equal to $\left(I_{p-1}, 0\right)$, where 0 is a vector of size $(p-1)$.

The term structure is given by the following proposition:

Proposition 12 : In the bivariate $\operatorname{SVARN}(p)$ Factor-Based Term Structure Model the price at date $t$ of the zero-coupon bond with residual maturity $h$ is :

$$
B(t, h)=\exp \left(C_{h}^{\prime} \tilde{X}_{t}+D_{h}^{\prime} Z_{t}\right), \text { for } h \geq 1
$$

where the vectors $C_{h}$ and $D_{h}$ satisfy the following recursive equations :

$$
\left\{\begin{array}{l}
C_{h}=\tilde{\Phi}^{*^{\prime}} C_{h-1}-c \\
D_{h}=-d+C_{1, h-1} \nu_{1}^{*^{\prime}}+C_{p+1, h-1} \nu_{2}^{*^{\prime}}+\frac{1}{2} C_{1, h-1}^{2}\left(\sigma_{1}^{* 2}+\varphi_{o}^{* 2} \sigma_{2}^{* 2}\right) \\
\quad+\left(C_{1, h-1}\right)\left(C_{p+1, h-1}\right) \varphi_{o}^{* 2} \sigma_{2}^{* 2}+\frac{1}{2} C_{p+1, h-1}^{2} \sigma_{2}^{* 2}+\tilde{D}_{h-1}+F\left(D_{1, h-1}\right),
\end{array}\right.
$$

where $\tilde{D}_{h-1}$ and $F\left(D_{1, h-1}\right)$ have the same meaning as in Proposition 8, and the initial conditions are $C_{0}=0, D_{0}=0$ (or $C_{1}=-c, D_{1}=-d$ ) [Proof : see Appendix 7].

So, Proposition 12 shows that the yields to maturity are:

$$
R(t, h)=-\frac{C_{h}^{\prime}}{h} \tilde{X}_{t}-\frac{D_{h}^{\prime}}{h} Z_{t}, \quad h \geq 1
$$

In the endogenous case we can take $x_{1 t}=r_{t+1}$, and $x_{2 t}=R(t, H)$ for a given time to maturity $H$. In this case the absence of arbitrage conditions for $r_{t+1}$ and $R(t, H)$ imply:

(i) $C_{1}=-e_{1}, D_{1}=0$, or $c=e_{1}, d=0$

(ii) $C_{H}=-H e_{p+1}, D_{H}=0$. 
Using the notations $C_{h}=\left(C_{1, h}, C_{1, h}^{*}, C_{p+1, h}, C_{2, h}^{*}\right)^{\prime}, \tilde{C}_{1, h}=\left(C_{1, h}^{*^{\prime}}, 0\right)^{\prime}, \tilde{C}_{2, h}=\left(C_{2, h}^{* \prime}, 0\right)^{\prime}$ (where the zeros are scalars), and $\tilde{C}_{h}=\left(\tilde{C}_{1, h}^{\prime}, \tilde{C}_{2, h}^{\prime}\right)^{\prime}$, it easily seen that the recursive equation $C_{h}=\tilde{\Phi}^{*^{\prime}} C_{h-1}-c$ can be written :

$$
C_{h}=\Phi_{1}^{*^{\prime}} C_{1, h-1}+\Phi_{2}^{*^{\prime}} C_{p+1, h-1}+\tilde{C}_{h-1}-c .
$$

Conditions $(i)$ are used as initial values in the recursive procedure of Proposition 10, and conditions (ii) implies restrictions on the parameters $\tilde{\Phi}^{*}, \nu_{1}^{*}, \nu_{2}^{*}$ which must be taken into account at the estimation stage. Note that, the number of constrains is less than the number of additional parameters appearing in the SDF and, therefore, the historical dynamics is not further constrained.

\subsection{Estimating SVARN $(p)$ Factor-Based Term Structure Models}

\subsubsection{Observable Factor Approach}

The purpose of this section is to propose an estimation methodology for the Gaussian term structure models presented above, in the case where the factor is a vector of yields at different maturities. The observable nature of the factor has several important advantages. First, thanks to data, we are able to detect stylized facts on interest rates which give us the possibility to justify the autoregressive model with switching regimes we propose for the historical dynamics of $\left(x_{t}\right)$ : indeed, a large empirical literature on bond yields show that interest rates have an historical multi-lag dynamics characterized by switching of regimes [see, among the others, Hamilton (1988), Garcia and Perron (1996), Christiansen and Lund (2005), Cochrane and Piazzesi (2005)]. Second, observations on the Gaussian-distributed factor lead to a maximum likelihood estimation of historical parameters and, therefore, we are able to rank the models in terms of various information criteria. Finally, the difference between directly observed and estimated factor values determine model residuals that can be used to derive various diagnostic criteria.

Compared with this (multi-lag regime-switching) observable factor approach, the classical continuous-time affine term structure approach à la Duffie and Kan (1996) and Dai and Singleton (2000) has some different features [see also Dai, Singleton and Yang (2006)]. First, the factors are in general assumed not observable and therefore justifications for the (historical) factors dynamics, along with a precise econometric analysis of model residuals, are not possible. Second, in order to reconstruct a time series of the latent factors, for an exact maximum likelihood estimation, prices of some zero-coupon bonds are assumed to be perfectly observed in order to inverse the pricing equations [see Chen and Scott (1993) and Pearson and Sun (1994)]; this inversion technique depends on the selected zero-coupon bonds and on their parameter values, which are not initially available, and therefore the reconstructed time series are model-sensitive [see also Collin-Dufresne, Goldstein and Jones (2005)]. Third, the class of Compound Autoregressive (Car) processes is much larger than the discrete-time counterpart of the continuous-time affine class ${ }^{9}$ [see Gourieroux, Monfort and Polimenis (2006), and Darolles, Gourieroux and Jasiak (2006)].

\subsubsection{Two-Step Estimation Procedure}

The methodology we follow to estimate the parameters of our (observable factor) Gaussian switching regime term structure models is based on a consistent two-step procedure. In the first step, using the observations on the endogenous factor $\left(x_{t}\right)$, the vector of historical parameters $\theta_{\mathbb{P}}$ is estimated

\footnotetext{
${ }^{9}$ For instance, the discrete-time Gaussian $\operatorname{VAR}(1)$ process has a continuous-time equivalent if and only if there exists a matrix $\vartheta \operatorname{such}$ that $\varphi=\exp (-\vartheta)$.
} 
by the maximization of the likelihood function calculated by means of the Kitagawa-Hamilton filter [see Hamilton (1994)]. In the second step, using observations on yields with maturities different from those used in the first step, and for a given estimates of the conditional historical (regimedependent) variance-covariance matrix, we estimate the vector of risk-neutral parameters $\theta_{\mathbb{Q}}$ by minimizing the sum of squared fitting errors between the observed and theoretical yields. The latent variable $Z_{t}=\left(z_{t}, \ldots, z_{t-p}\right)^{\prime}$, in the yield-to-maturity formula of the $\operatorname{SVARN}(p)$ model, is extracted using smoothed probabilities, for each regime and each date, calculated with a generalization of the Kim's smoothing algorithm [see Appendix 8 for the proof, and Billio and Monfort (1998) for an equivalent result in a Kalman filtering setting]. Thus, we estimate $\theta_{\mathbb{Q}}$ by nonlinear least squares (NLLS) constrained by restrictions (69)-(ii) implied by the absence of arbitrage opportunity on the long rate.

We denote by $H^{*}$ the set of maturities (except the short rate) used to estimate $\theta_{\mathbb{P}}$, and by $H^{* *}$ the set of remaining maturities used to estimate the vector of risk-neutral parameters $\theta_{\mathbb{Q}}$ (different from the historical parameters). The constrained NLLS estimator is given by :

$$
\left\{\begin{array}{l}
\hat{\theta}_{\mathbb{Q}}=\operatorname{Arg} \min _{\theta_{\mathbb{Q}}} S^{2}\left(\theta_{\mathbb{Q}}\right) \\
S^{2}\left(\theta_{\mathbb{Q}}\right)=\sum_{t=p}^{T} \sum_{h \in H^{* *}}[\tilde{R}(t, h)-R(t, h)]^{2}, \\
\text { s. t. } \sum_{t=p}^{T} \sum_{h \in H^{*}}[\tilde{R}(t, h)-R(t, h)]^{2}=0,
\end{array}\right.
$$

where $\tilde{R}(t, h)$ and $R(t, h)$ denote, respectively, the observed and model-implied yields in which $\theta_{\mathbb{P}}$ has been replaced the maximum likelihood estimator and $Z_{t}$ by smoothed values. The constraints in the minimization program (70) guarantees the absence of arbitrage opportunity on the yields determining the factor $\left(x_{t}\right)$, with the exception of the short rate for which the arbitrage restriction is automatically satisfied.

\section{Switching Autoregressive Gamma (SARG) Factor-Based Term Structure Model of order $p$}

Like for $\operatorname{SARN}(p)$ models, we start the description of the $\operatorname{SARG}(p)$ modeling by the case of one latent factor.

\subsection{The historical dynamics}

We assume that the Laplace transform of the conditional distribution of $x_{t+1}$, given $\left(\underline{x_{t}}, \underline{z_{t}}\right)$, is:

$$
\begin{gathered}
E\left[\exp \left(u x_{t+1}\right) \mid \underline{x_{t}},, \underline{z_{t}}\right]=\exp \left[\frac{u}{1-u \mu\left(Z_{t}, X_{t}\right)}\left[\varphi_{1}\left(Z_{t}\right) x_{t}+\ldots+\varphi_{p}\left(Z_{t}\right) x_{t-p+1}\right]\right. \\
\left.-\nu\left(Z_{t}\right) \log \left(1-u \mu\left(Z_{t}, X_{t}\right)\right)\right]
\end{gathered}
$$

where $Z_{t}=\left(z_{t}^{\prime}, \ldots, z_{t-p}^{\prime}\right)^{\prime}$, with $z_{t}$ a $J$-state non-homogeneous Markov chain such that $P\left(z_{t+1}=\right.$ 
$\left.e_{j} \mid z_{t}=e_{i} ; \underline{x_{t}}\right)=\pi\left(e_{i}, e_{j} ; \tilde{X}_{t}\right)$, and where $X_{t}=\left(x_{t}, \ldots, x_{t+1-p}\right)^{\prime}$. Using the notation:

$$
\begin{aligned}
A\left[u ; \varphi\left(Z_{t}\right), \mu\left(Z_{t}, X_{t}\right)\right] & =\frac{u}{1-u \mu\left(Z_{t}, X_{t}\right)}\left[\varphi_{1}\left(Z_{t}\right), \ldots, \varphi_{p}\left(Z_{t}\right)\right]^{\prime}=\frac{u}{1-u \mu\left(Z_{t}, X_{t}\right)} \varphi\left(Z_{t}\right) \\
b\left[u ; \nu\left(Z_{t}\right), \mu\left(Z_{t}, X_{t}\right)\right] & =-\nu\left(Z_{t}\right) \log \left(1-u \mu\left(Z_{t}, X_{t}\right)\right),
\end{aligned}
$$

relation (71) can be written:

$$
E\left[\exp \left(u x_{t+1}\right) \mid \underline{x_{t}}, \underline{z_{t}}\right]=\exp \left\{A\left[u ; \varphi\left(Z_{t}\right), \mu\left(Z_{t}, X_{t}\right)\right]^{\prime} X_{t}+b\left[u ; \nu\left(Z_{t}\right), \mu\left(Z_{t}, X_{t}\right)\right]\right\} .
$$

The process $\left(x_{t}\right)$ can also be written:

$$
\begin{aligned}
x_{t+1} & =\nu\left(Z_{t}\right) \mu\left(Z_{t}, X_{t}\right)+\varphi_{1}\left(Z_{t}\right) x_{t}+\ldots+\varphi_{p}\left(Z_{t}\right) x_{t+1-p}+\varepsilon_{t+1} \\
& =\nu\left(Z_{t}\right) \mu\left(Z_{t}, X_{t}\right)+\varphi\left(Z_{t}\right)^{\prime} X_{t}+\varepsilon_{t+1},
\end{aligned}
$$

where $\varepsilon_{t+1}$ is a martingale difference sequence with conditional Laplace transform given by:

$$
\begin{aligned}
E\left[\exp \left(u \varepsilon_{t+1}\right) \mid \underline{x_{t}}, \underline{\left.z_{t}\right]=} \exp \{-\right. & u\left[\nu\left(Z_{t}\right) \mu\left(Z_{t}, X_{t}\right)+\varphi\left(Z_{t}\right)^{\prime} X_{t}\right] \\
& \left.+A\left[u ; \varphi\left(Z_{t}\right), \mu\left(Z_{t}, X_{t}\right)\right]^{\prime} X_{t}+b\left[u ; \nu\left(Z_{t}\right), \mu\left(Z_{t}, X_{t}\right)\right]\right\} \\
=\exp \{[ & \left.A\left[u ; \varphi\left(Z_{t}\right), \mu\left(Z_{t}, X_{t}\right)\right]-u \varphi\left(Z_{t}\right)\right]^{\prime} X_{t} \\
& \left.+b\left[u ; \nu\left(Z_{t}\right), \mu\left(Z_{t}, X_{t}\right)\right]-u \nu\left(Z_{t}\right) \mu\left(Z_{t}, X_{t}\right)\right\} .
\end{aligned}
$$

Note that the dynamics of $\left(x_{t}, z_{t}\right)$ is in general not Car.

\subsection{The Stochastic Discount Factor}

In the $\operatorname{SARG}(p)$ model the $\mathrm{SDF}$ is specified in the following way:

$$
\begin{gathered}
M_{t, t+1}=\exp \left\{-c^{\prime} X_{t}-d^{\prime} Z_{t}+\Gamma\left(Z_{t}, X_{t}\right) \varepsilon_{t+1}+\Gamma\left(Z_{t}, X_{t}\right)\left[\nu\left(Z_{t}\right) \mu\left(Z_{t}, X_{t}\right)+\varphi\left(Z_{t}\right)^{\prime} X_{t}\right]\right. \\
-A\left[\Gamma\left(Z_{t}, X_{t}\right) ; \varphi\left(Z_{t}\right), \mu\left(Z_{t}, X_{t}\right)\right]^{\prime} X_{t} \\
\left.\quad-b\left[\Gamma\left(Z_{t}, X_{t}\right) ; \nu\left(Z_{t}\right), \mu\left(Z_{t}, X_{t}\right)\right]-\delta\left(Z_{t}, X_{t}\right)^{\prime} z_{t+1}\right\},
\end{gathered}
$$

where $\Gamma\left(Z_{t}, X_{t}\right)=\gamma\left(Z_{t}\right)+\tilde{\gamma}^{\prime}\left(Z_{t}\right) X_{t}$, or, equivalently

$$
\begin{gathered}
M_{t, t+1}=\exp \left\{-c^{\prime} X_{t}-d^{\prime} Z_{t}+\Gamma\left(Z_{t}, X_{t}\right) x_{t+1}-A\left[\Gamma\left(Z_{t}, X_{t}\right) ; \varphi\left(Z_{t}\right), \mu\left(Z_{t}, X_{t}\right)\right]^{\prime} X_{t}\right. \\
\left.-b\left[\Gamma\left(Z_{t}, X_{t}\right) ; \nu\left(Z_{t}\right), \mu\left(Z_{t}, X_{t}\right)\right]-\delta\left(Z_{t}, X_{t}\right)^{\prime} z_{t+1}\right\}
\end{gathered}
$$

Assuming the normalisation condition (44), we get that:

$$
r_{t+1}=c^{\prime} X_{t}+d^{\prime} Z_{t} .
$$




\subsection{Useful Lemmas}

In the subsequent sections we will use several times the following lemmas. Let us consider the functions:

$$
\tilde{a}(u ; \rho, \mu)=\frac{\rho u}{1-u \mu} \text { and } \tilde{b}(u ; \nu, \mu)=-\nu \log (1-u \mu) ;
$$

we have:

\section{Lemma 1 :}

$$
\begin{aligned}
& \tilde{a}(u+\alpha ; \rho, \mu)-\tilde{a}(\alpha ; \rho, \mu)=\tilde{a}\left(u ; \rho^{*}, \mu^{*}\right) \\
& \tilde{b}(u+\alpha ; \nu, \mu)-\tilde{b}(\alpha ; \nu, \mu)=\tilde{b}\left(u ; \nu, \mu^{*}\right) \\
& \text { with } \rho^{*}=\frac{\rho}{(1-\alpha \mu)^{2}}, \mu^{*}=\frac{\mu}{1-\alpha \mu},
\end{aligned}
$$

[Proof : see Appendix 9].

Lemma 1 immediately implies Lemma 2.

Lemma 2 :

$$
\begin{aligned}
& A\left[u+\alpha ; \varphi\left(Z_{t}\right), \mu\left(Z_{t}, X_{t}\right)\right]-A\left[\alpha ; \varphi\left(Z_{t}\right), \mu\left(Z_{t}, X_{t}\right)\right]=A\left[u ; \varphi^{*}\left(Z_{t}\right), \mu^{*}\left(Z_{t}, X_{t}\right)\right] \\
& b\left[u+\alpha ; \nu\left(Z_{t}\right), \mu\left(Z_{t}, X_{t}\right)\right]-b\left[\alpha ; \nu\left(Z_{t}\right), \mu\left(Z_{t}, X_{t}\right)\right]=b\left[u ; \nu\left(Z_{t}\right), \mu^{*}\left(Z_{t}, X_{t}\right)\right] \\
& \text { with } \varphi^{*}\left(Z_{t}\right)=\frac{\varphi\left(Z_{t}\right)}{\left[1-\alpha \mu\left(Z_{t}, X_{t}\right)\right]^{2}}, \mu^{*}\left(Z_{t}, X_{t}\right)=\frac{\mu\left(Z_{t}, X_{t}\right)}{1-\alpha \mu\left(Z_{t}, X_{t}\right)} .
\end{aligned}
$$

\subsection{Risk-neutral dynamics}

The Laplace transform of the risk-neutral conditional distribution of $\left(x_{t+1}, z_{t+1}\right)$ is, using the notation $\Gamma_{t}=\Gamma\left(X_{t}, Z_{t}\right)$ :

$$
\begin{array}{r}
E_{t}^{\mathbb{Q}}\left[\exp \left(u x_{t+1}+v^{\prime} z_{t+1}\right)\right]=E_{t}\left\{\operatorname { e x p } \left[\left(u+\Gamma_{t}\right) x_{t+1}-A\left[\Gamma_{t} ; \varphi\left(Z_{t}\right), \mu\left(Z_{t}, X_{t}\right)\right]^{\prime} X_{t}\right.\right. \\
\left.\left.-b\left[Z_{t} ; \nu\left(Z_{t}\right), \mu\left(Z_{t}, X_{t}\right)\right]+\left(v-\delta\left(Z_{t}, X_{t}\right)\right)^{\prime} z_{t+1}\right]\right\} \\
=\exp \left\{\left[\left(A\left[u+\Gamma_{t} ; \varphi\left(Z_{t}\right), \mu\left(Z_{t}, X_{t}\right)\right]-A\left[\Gamma_{t} ; \varphi\left(Z_{t}\right), \mu\left(Z_{t}, X_{t}\right)\right]\right)^{\prime} X_{t}\right.\right. \\
\left.\left.+b\left[u+\Gamma_{t} ; \nu\left(Z_{t}\right), \mu\left(Z_{t}, X_{t}\right)\right]-b\left[\Gamma_{t} ; \nu\left(Z_{t}\right), \mu\left(Z_{t}, X_{t}\right)\right]\right]\right\} \\
\times \sum_{j=1}^{J} \pi\left(z_{t}, e_{j} ; X_{t}\right) \exp \left[\left(v-\delta\left(Z_{t}, X_{t}\right)\right)^{\prime} e_{j}\right],
\end{array}
$$

and, using Lemma 2, (79) can be written:

$$
\begin{aligned}
E_{t}^{\mathbb{Q}}\left[\exp \left(u x_{t+1}+v^{\prime} z_{t+1}\right)\right]=\exp \left\{A\left[u ; \varphi^{*}\left(Z_{t}\right), \mu^{*}\left(Z_{t}, X_{t}\right)\right]^{\prime} X_{t}+b\left[u ; \nu\left(Z_{t}\right), \mu^{*}\left(Z_{t}, X_{t}\right)\right]\right\} \\
\times \sum_{j=1}^{J} \pi\left(z_{t}, e_{j} ; X_{t}\right) \exp \left[\left(v-\delta\left(Z_{t}, X_{t}\right)\right)^{\prime} e_{j}\right]
\end{aligned}
$$

with $\varphi^{*}\left(Z_{t}\right)=\frac{\varphi\left(Z_{t}\right)}{\left[1-\Gamma_{t} \mu\left(Z_{t}, X_{t}\right)\right]^{2}}$ and $\mu^{*}\left(Z_{t}, X_{t}\right)=\frac{\mu\left(Z_{t}, X_{t}\right)}{1-\Gamma_{t} \mu\left(Z_{t}, X_{t}\right)}$. 
So, from (72), we see that the risk-neutral conditional distribution of $x_{t+1}$, given $\left(x_{t}, z_{t}\right)$, is in the same class as the historical one and obtained by replacing $\varphi\left(Z_{t}\right)$ with $\varphi^{*}\left(Z_{t}\right)$, and $\bar{\mu}\left(Z_{t}, X_{t}\right)$ with $\mu^{*}\left(Z_{t}, X_{t}\right)$.

In order to get a generalize linear term structure we impose that the risk-neutral dynamics is a switching regime Gamma $\operatorname{Car}(p)$ process. So, using the results in Section 2.5.b, we get that $\varphi^{*}\left(Z_{t}\right)$ and $\mu^{*}\left(Z_{t}, X_{t}\right)$ must be constant, $\nu\left(Z_{t}\right)=\nu^{*^{\prime}} Z_{t}$ and $\pi\left(z_{t}, e_{j} ; X_{t}\right)=\pi^{*}\left(z_{t}, e_{j}\right) \exp \left[\left(\delta\left(Z_{t}, X_{t}\right)\right)^{\prime} e_{j}\right]$. Also note that $\mu^{*}$ must be positive as well as the components of $\nu^{*}$ and $\varphi^{*}$. This implies the following constraint on the historical dynamics and on the SDF:

$$
\begin{array}{ll}
\mu\left(Z_{t}, X_{t}\right) & =\mu^{*}\left[1-\Gamma\left(Z_{t}, X_{t}\right) \mu\left(Z_{t}, X_{t}\right)\right] \\
\varphi\left(Z_{t}\right) & =\varphi^{*}\left[1-\Gamma\left(Z_{t}, X_{t}\right) \mu\left(Z_{t}, X_{t}\right)\right]^{2} \\
\nu\left(Z_{t}\right) & =\nu^{*^{\prime}} Z_{t} \\
\delta_{j}\left(Z_{t}, X_{t}\right) & =\log \left[\frac{\pi\left(z_{t}, e_{j} ; X_{t}\right)}{\pi^{*}\left(z_{t}, e_{j}\right)}\right] .
\end{array}
$$

We see that $\varphi\left(Z_{t}\right)=\frac{\varphi^{*}}{\mu^{* 2}} \mu\left(Z_{t}, X_{t}\right)^{2}$, so $\mu\left(Z_{t}, X_{t}\right)$ must depend only on $Z_{t}$, and therefore the same is true for $\Gamma\left(Z_{t}, X_{t}\right)$. Finally, we have the constraint:

i)

$$
\mu\left(Z_{t}\right)=\mu^{*}\left[1-\Gamma\left(Z_{t}\right) \mu\left(Z_{t}\right)\right]
$$

ii)

$$
\varphi\left(Z_{t}\right)=\varphi^{*}\left[1-\Gamma\left(Z_{t}\right) \mu\left(Z_{t}\right)\right]^{2}
$$

iii)

$$
\nu\left(Z_{t}\right)=\nu^{*^{\prime}} Z_{t}
$$

iv)

$$
\delta_{j}\left(Z_{t}, X_{t}\right)=\log \left[\frac{\pi\left(z_{t}, e_{j} ; X_{t}\right)}{\pi^{*}\left(z_{t}, e_{j}\right)}\right]
$$

In particular, since $\varphi\left(Z_{t}\right)=\frac{\varphi^{*}}{\mu^{* 2}} \mu\left(Z_{t}\right)^{2}$, the random vector must be proportional to a deterministic vector.

Moreover, it is easily seen that the risk premium corresponding to the payoff $\exp \left(-\theta x_{t+1}\right)$ at $t+1$ is:

$$
\begin{aligned}
\omega_{t}(\theta)=\{A[-\theta & \left.\left.; \varphi\left(Z_{t}\right), \mu\left(Z_{t}\right)\right]-A\left[-\theta ; \varphi^{*}, \mu^{*}\right]\right\}^{\prime} X_{t} \\
& +b\left[-\theta ; \nu^{*^{\prime}} Z_{t}, \mu\left(Z_{t}\right)\right]-b\left[-\theta ; \nu^{*^{\prime}} Z_{t}, \mu^{*}\right] .
\end{aligned}
$$

Like in the gaussian case, we obtain an affine function in $X_{t}$ also depending on $Z_{t}$. The risk premium associated with the digital asset providing one money unit at $t+1$ if $z_{t+1}=e_{j}$, is still given by (47). 


\subsection{The Generalised Linear Term Structure}

Let us introduce the notations:

$$
\begin{aligned}
& A^{*}(u)=A\left(u ; \varphi^{*}, \mu^{*}\right) \\
& \tilde{C}_{h}=\left(C_{2, h}, \ldots, C_{p, h}, 0\right)^{\prime} .
\end{aligned}
$$

As usual, $B(t, h)$ is the price at $t$ of a zero-coupon bond with residual maturity $h$.

Proposition 13 : In the univariate $\operatorname{SARG}(p)$ Factor-Based Term Structure Model the price at date $t$ of the zero-coupon bond with residual maturity $h$ is :

$$
B(t, h)=\exp \left(C_{h}^{\prime} X_{t}+D_{h}^{\prime} Z_{t}\right), \text { for } h \geq 1,
$$

where the vectors $C_{h}$ and $D_{h}$ satisfy the following recursive equations :

$$
\left\{\begin{array}{l}
C_{h}=-c+A^{*}\left(C_{1, h-1}\right)+\tilde{C}_{h-1} \\
D_{h}=-d-\nu^{*} \log \left(1-C_{1, h-1} \mu^{*}\right)+\tilde{D}_{h-1}+F\left(D_{1, h-1}\right),
\end{array}\right.
$$

where $\tilde{D}_{h-1}$ and $F\left(D_{1, h-1}\right)$ have the same meaning as in Proposition 8; the initial conditions are $C_{0}=0, D_{0}=0$ (or $\left.C_{1}=-c, D_{1}=-d\right)$ [Proof : see Appendix 10].

Again, we obtain a generalised linear term structure given by:

$$
R(t, h)=-\frac{C_{h}^{\prime}}{h} X_{t}-\frac{D_{h}^{\prime}}{h} Z_{t}, \quad h \geq 1
$$

and, in the same spirit of Propositions 9 and 10 for the univariate $\operatorname{SARN}(p)$ model [see Section 3.6], it is easy to verify that the processes $R=[R(t, h), 0 \leq t<T]$ and $R_{\mathcal{H}}=[R(t, h), 0 \leq t<T, h \in$ $\mathcal{H}]$ are, respectively, a weak Switching $\operatorname{ARMA}(p, p-1)$ process and a weak $H$-variate Switching $\operatorname{VARMA}(p, p-1)$ process.

In the endogenous case, where $x_{t}=r_{t+1}$, the previous results remains valid with $C_{1}=-e_{1}, D_{1}=0$.

\subsection{Positivity of the yields}

Since $r_{t+1}=R(t, 1)=c^{\prime} X_{t}+d^{\prime} Z_{t}$, and since the components of $X_{t}$ are positive, the short term process will be positive as soon as the components of $c$ and $d$ are nonnegative. The positivity of $r_{t+1}$ implies that of $R(t, h)$, at any date $t$ and time to maturity $h$, because $R(t, h)=$ $-\frac{1}{h} \log E_{t}^{\mathbb{Q}}\left[\exp \left(-r_{t+1}-\ldots-r_{t+h}\right)\right]$.

This positivity can also be observed from the recursive equations of Proposition 13. Indeed, using the fact that $\mu^{*}$ and the components of $\varphi^{*}$ and $\nu^{*}$ are positive and that $0<\pi_{i j}^{*}<1$, it easily seen that, for any $u<0$, the components of $A^{*}(u)$ and $-\nu^{*} \log \left(1-C_{1, h-1} \mu^{*}\right)$ are negative and the result follows. 


\subsection{Multi-Factor generalization : the SVARG $(p)$ Factor-Based Term Structure Model}

The bivariate process $\tilde{x_{t}}=\left(x_{1, t}, x_{2, t}\right)$ is a $\operatorname{SVARG}(p)$ model defined by the following conditional Laplace transforms:

$$
\begin{gathered}
E_{t}\left[\exp \left(u_{1} x_{1, t+1}\right) \mid \underline{x_{2, t+1}}, \underline{x_{1, t}}, \underline{z_{t}}\right]=\exp \left\{\frac{u_{1}}{1-u_{1} \mu_{1}\left(Z_{t}\right)}\left[\varphi_{o}\left(Z_{t}\right) x_{2, t+1}+\varphi_{11}\left(Z_{t}\right)^{\prime} X_{1 t}+\varphi_{12}\left(Z_{t}\right)^{\prime} X_{2 t}\right]\right. \\
\left.-\nu_{1}\left(Z_{t}\right) \log \left(1-u_{1} \mu_{1}\left(Z_{t}\right)\right)\right\} \\
E_{t}\left[\exp \left(u_{2} x_{2, t+1}\right) \underline{\mid x_{1, t}}, \underline{x_{2, t}}, \underline{z_{t}}\right]=\exp \left\{\frac{u_{2}}{1-u_{2} \mu_{2}\left(Z_{t}\right)}\left[\varphi_{21}\left(Z_{t}\right)^{\prime} X_{1 t}+\varphi_{22}\left(Z_{t}\right)^{\prime} X_{2 t}\right]\right. \\
\left.-\nu_{2}\left(Z_{t}\right) \log \left(1-u_{2} \mu_{2}\left(Z_{t}\right)\right)\right\}
\end{gathered}
$$

We will use the notations:

$$
\begin{aligned}
& \varphi_{o}\left(Z_{t}\right)=\varphi_{o, t}, \\
& {\left[\varphi_{11}\left(Z_{t}\right)^{\prime}, \varphi_{12}\left(Z_{t}\right)^{\prime}\right]=\varphi_{1, t}^{\prime}, \quad\left[\varphi_{21}\left(Z_{t}\right)^{\prime}, \varphi_{22}\left(Z_{t}\right)^{\prime}\right]=\varphi_{2, t}^{\prime},} \\
& \mu_{i}\left(Z_{t}\right)=\mu_{i, t}, \quad \nu_{i}\left(Z_{t}\right)=\nu_{i, t}, \quad i \in\{1,2\},
\end{aligned}
$$

and using the functions $\tilde{a}, \tilde{b}, A, B$ defined in Lemma 1 and in Section 4.1, we will introduce the notations:

$$
\begin{aligned}
& a_{1, t}\left(u_{1}\right)=\tilde{a}\left(u_{1} ; \varphi_{o, t}, \mu_{1, t}\right) \\
& b_{1, t}\left(u_{1}\right)=\tilde{b}\left(u_{1} ; \nu_{1, t}, \mu_{1, t}\right), \quad b_{2, t}\left(u_{2}\right)=\tilde{b}\left(u_{2} ; \nu_{2, t}, \mu_{2, t}\right) \\
& A_{1, t}\left(u_{1}\right)=A\left(u_{1} ; \varphi_{1, t}, \mu_{1, t}\right), A_{2, t}\left(u_{2}\right)=A\left(u_{2} ; \varphi_{2, t}, \mu_{2, t}\right) .
\end{aligned}
$$

With these notations, the Laplace transforms (84) and (85) become respectively:

$$
\begin{gathered}
E_{t}\left[\exp \left(u_{1} x_{1, t+1}\right) \mid \underline{x_{2, t+1}}, \underline{x_{1, t}}, \underline{z_{t}}\right]=\exp \left[a_{1, t}\left(u_{1}\right) x_{2, t+1}+A_{1, t}\left(u_{1}\right)^{\prime} \tilde{X}_{t}+b_{1, t}\left(u_{1}\right)\right] \\
E_{t}\left[\exp \left(u_{2} x_{2, t+1}\right) \mid \underline{x_{1, t}}, \underline{x_{2, t}}, \underline{z_{t}}\right]=\exp \left[A_{2, t}\left(u_{2}\right)^{\prime} \tilde{X}_{t}+b_{2, t}\left(u_{2}\right)\right]
\end{gathered}
$$

where $\tilde{X}_{t}=\left(X_{1 t}^{\prime}, X_{2 t}^{\prime}\right)^{\prime}$. Moreover, the joint conditional Laplace transform of $\left(x_{1, t+1}, x_{2, t+1}\right)$, given $\left(\underline{x_{1, t}}, \underline{x_{2, t}}, \underline{z_{t}}\right)$, is:

$$
\begin{aligned}
& E_{t}\left[\exp \left(u_{1} x_{1, t+1}+u_{2} x_{2, t+1}\right) \mid \underline{x_{1, t}}, \underline{x_{2, t}}, \underline{z_{t}}\right] \\
= & \exp \left\{\left[A_{1, t}\left(u_{1}\right)+A_{2, t}\left(u_{2}+a_{1, t}\left(u_{1}\right)\right)\right]^{\prime} \tilde{X}_{t}+b_{1, t}\left(u_{1}\right)+b_{2, t}\left(u_{2}+a_{1, t}\left(u_{1}\right)\right)\right\} .
\end{aligned}
$$

The process $z_{t}$ is assumed to be a non-homogeneous Markov chain such that $P\left(z_{t+1}=e_{j} \mid z_{t}=\right.$ $\left.e_{i} ; \underline{\tilde{x}_{t}}\right)=\pi\left(e_{i}, e_{j} ; \tilde{X}_{t}\right)$. 
We now introduce the SDF:

$$
\begin{aligned}
M_{t, t+1}=\exp \left\{-c^{\prime} \tilde{X}_{t}-d^{\prime} Z_{t}+\Gamma_{1 t} x_{1, t+1}+\Gamma_{2 t} x_{2, t+1}\right. \\
-\left[A_{1, t}\left(\Gamma_{1 t}\right)+A_{2, t}\left(\Gamma_{2 t}+a_{1, t}\left(\Gamma_{1 t}\right)\right)\right]^{\prime} \tilde{X}_{t} \\
\left.-\left[b_{1, t}\left(\Gamma_{1 t}\right)+b_{2, t}\left(\Gamma_{2 t}+a_{1, t}\left(\Gamma_{1 t}\right)\right)\right]-\delta\left(Z_{t}, \tilde{X}_{t}\right)^{\prime} z_{t+1}\right\},
\end{aligned}
$$

where $\Gamma_{1 t}=\Gamma_{1}\left(Z_{t}\right)$ and $\Gamma_{2 t}=\Gamma_{2}\left(Z_{t}\right)$.

\subsection{Risk-neutral dynamics in the multifactor case}

We can now present, using the lemmas presented above, the joint conditional Laplace transform of $\left(x_{1, t+1}, x_{2, t+1}\right)$ in the risk-neutral world in the following proposition.

Proposition 14 : The joint conditional Laplace transform of $\left(x_{1, t+1}, x_{2, t+1}\right)$ in the risk-neutral world is given by :

$$
\begin{aligned}
E_{t}^{\mathbb{Q}}\left[\exp \left(u_{1} x_{1, t+1}+u_{2} x_{2, t+1}\right) \mid \underline{x_{1 t}}, \underline{x_{2 t}}, \underline{z_{t}}\right]= & \exp \left\{\left[A_{1, t}^{*}\left(u_{1}\right)+A_{2, t}^{*}\left[u_{2}+a_{1, t}^{*}\left(u_{1}\right)\right]\right]^{\prime} \tilde{X}_{t}\right. \\
& \left.+b_{2, t}^{*}\left[u_{2}+a_{1, t}^{*}\left(u_{1}\right)\right]+b_{1, t}^{*}\left(u_{1}\right)\right\},
\end{aligned}
$$

where

$$
\begin{array}{ll}
A_{1, t}^{*}\left(u_{1}\right) & =A_{1}\left(u_{1} ; \varphi_{1 t}^{*}, \mu_{1 t}^{*}\right), \\
A_{2, t}^{*}\left[u_{2}+a_{1, t}^{*}\left(u_{1}\right)\right] & =A\left[u_{2}+\tilde{a}\left(u_{1} ; \varphi_{o t}^{*}, \mu_{1, t}^{*}\right) ; \varphi_{2 t}^{*}, \mu_{2 t}^{*}\right], \\
b_{2, t}^{*}\left[u_{2}+a_{1, t}^{*}\left(u_{1}\right)\right] & =\tilde{b}\left[u_{2}+\tilde{a}\left(u_{1} ; \varphi_{o t}^{*}, \mu_{1, t}^{*}\right) ; \nu_{2 t}^{*}, \mu_{2 t}^{*}\right], \\
b_{1, t}^{*}\left(u_{1}\right) & =\tilde{b}_{1}\left(u_{1} ; \nu_{1 t}^{*}, \mu_{1 t}^{*}\right),
\end{array}
$$

and with

$$
\begin{aligned}
& \varphi_{o t}^{*}=\frac{\varphi_{o t}}{\left(1-\Gamma_{1 t} \mu_{1 t}\right)^{2}}, \quad \varphi_{1 t}^{*}=\frac{\varphi_{1 t}}{\left(1-\Gamma_{1 t} \mu_{1 t}\right)^{2}}, \quad \varphi_{2 t}^{*}=\frac{\varphi_{2 t}}{\left\{1-\left[\Gamma_{2 t}+a_{1, t}\left(\Gamma_{1 t}\right)\right] \mu_{2 t}\right\}^{2}} \\
& \mu_{1 t}^{*}=\frac{\mu_{1 t}}{\left(1-\Gamma_{1 t} \mu_{1 t}\right)}, \quad \mu_{2 t}^{*}=\frac{\mu_{2 t}}{\left\{1-\left[\Gamma_{2 t}+a_{1, t}\left(\Gamma_{1 t}\right)\right] \mu_{2 t}\right\}} .
\end{aligned}
$$

So, (90) has exactly the same form as (88) with different parameters. In other words the risk-neutral dynamics belongs to the same class as the historical one [Proof : see Appendix 11].

In order to have a Car process in the risk-neutral world, we know from Section 2.9 that we must have the following constraint between the SDF and the historical dynamics:

i)

$$
\frac{\mu_{1 t}}{1-\Gamma_{1 t} \mu_{1 t}}=\mu_{1}^{*}
$$

ii)

$$
\frac{\varphi_{1 t}}{\left(1-\Gamma_{1 t} \mu_{1 t}\right)^{2}}=\varphi_{1}^{*}
$$


iii)

$$
\nu_{1}\left(Z_{t}\right)=\nu_{1}^{*^{\prime}} Z_{t}
$$

iv)

$$
\frac{\varphi_{o t}}{\left(1-\Gamma_{1 t} \mu_{1 t}\right)^{2}}=\varphi_{o}^{*}
$$

v)

$$
\frac{\mu_{2 t}}{1-\left[\Gamma_{2 t}+a_{1, t}\left(\Gamma_{1 t}\right)\right] \mu_{2 t}}=\mu_{2}^{*}
$$

vi)

$$
\frac{\varphi_{2 t}}{\left(1-\left[\Gamma_{2 t}+a_{1, t}\left(\Gamma_{1 t}\right)\right] \mu_{2 t}\right)^{2}}=\varphi_{2}^{*}
$$

vii)

$$
\nu_{2}\left(Z_{t}\right)=\nu_{2}^{*^{\prime}} Z_{t}
$$

Moreover, the constraint on the dynamics of the Markov chain are the same as in the gaussian case, namely:

viii)

$$
\delta_{j}\left(Z_{t}, \tilde{X}_{t}\right)=\log \left[\frac{\pi\left(z_{t}, e_{j} ; \tilde{X}_{t}\right)}{\pi^{*}\left(z_{t}, e_{j}\right)}\right] .
$$

It is worth noting that, if there is no instantaneous causality between $x_{1, t+1}$ and $x_{2, t+1}$, that is if $\varphi_{o t}=0$, function $a_{1 t}$ is also equal to zero and constraint $v$ ) and $v i$ ) are simpler and become similar to $i$ ) and $i i$.

\subsection{The Generalized Linear Term Structure in the multifactor case}

Using the notations:

$$
\begin{aligned}
& a_{1}^{*}\left(u_{1}\right)=\tilde{a}\left(u_{1} ; \varphi_{o}^{*}, \mu_{1}^{*}\right), \\
& A_{1}^{*}\left(u_{1}\right)=A\left(u_{1} ; \varphi_{1}^{*}, \mu_{1}^{*}\right), \\
& A_{2}^{*}\left(u_{2}\right)=A\left(u_{2} ; \varphi_{2}^{*}, \mu_{2}^{*}\right), \\
& \tilde{C}_{h}=\left(C_{2, h}, \ldots, C_{p, h}, 0, C_{p+2, h}, \ldots, C_{2 p, h}, 0\right)^{\prime},
\end{aligned}
$$

we have

Proposition 15 : In the bivariate $\operatorname{SVARG}(p)$ Factor-Based Term Structure Model the price at date $t$ of the zero-coupon bond with residual maturity $h$ is :

$$
B(t, h)=\exp \left(C_{h}^{\prime} \tilde{X}_{t}+D_{h}^{\prime} Z_{t}\right), \text { for } h \geq 1
$$


where the vectors $C_{h}$ and $D_{h}$ satisfy the following recursive equations :

$$
\left\{\begin{array}{c}
C_{h}=-c+A_{1}^{*}\left(C_{1, h-1}\right)+A_{2}^{*}\left[C_{p+1, h-1}+a_{1}^{*}\left(C_{1, h-1}\right)\right]+\tilde{C}_{h-1} \\
D_{h}=-d-\nu_{1}^{*} \log \left(1-C_{1, h-1} \mu_{1}^{*}\right)-\nu_{2}^{*} \log \left[1-\left(C_{p+1, h-1}+a_{1}^{*}\left(C_{1, h-1}\right)\right) \mu_{2}^{*}\right] \\
+\tilde{D}_{h-1}+F\left(D_{1, h-1}\right)
\end{array}\right.
$$

where $\tilde{D}_{h-1}$ and $F\left(D_{1, h-1}\right)$ have the same meaning as in Proposition 8; the initial conditions are $C_{0}=0, D_{0}=0$ (or $C_{1}=-c, D_{1}=-d$ ) [Proof : see Appendix 12].

So, Proposition 15 shows that, also for the SVARG $(p)$ model, yields to maturity are linear functions of $\tilde{X}_{t}$ and $Z_{t}$.

In the endogenous case, we can consider as factors the short rate $r_{t+1}$ and the long rate $R(t, H)$, for a given time to maturity $H$. Now, if we want to define a joint historical and risk-neutral dynamics for these variables, compatible with the no-arbitrage opportunity condition, we have to take into account domain restrictions on $R(t, H)$ : given that the support of $r_{t+1}$ is $\mathcal{D}_{1}=(0,+\infty)$, under A.A.O. the support of $R(t, H)$ has to be $\mathcal{D}_{H}=[b,+\infty)$, for some constant $b>0$ [see Gourieroux, Monfort (2006b) for details]. Consequently, the bivariate $\operatorname{SVARG}(p)$ process $\tilde{x}_{t}$, being with support $\mathcal{D}=\mathcal{D}_{1} \times \mathcal{D}_{1}$, will be specified for $x_{1 t}=r_{t+1}$ and $x_{2 t}=R(t, H)-b$, and the results presented for the $\operatorname{SVARN}(p)$ case [see Section 3.8] will apply also in this case.

It is also easily seen that the risk premium of the payoff $p_{t+1}=\exp \left(-\theta_{1} x_{1, t+1}-\theta_{2} x_{2, t+1}\right)$ is:

$$
\begin{aligned}
\omega_{t}\left(\theta_{1}, \theta_{2}\right)=\left\{A_{2, t}[\right. & \left.\left.-\theta_{2}+a_{1, t}\left(-\theta_{1}\right)\right]+A_{1, t}\left(-\theta_{1}\right)-A_{2}^{*}\left[-\theta_{2}+a_{1}^{*}\left(-\theta_{1}\right)\right]-A_{1}^{*}\left(-\theta_{1}\right)\right\}^{\prime} X_{t} \\
& +b_{2, t}\left[-\theta_{2}+a_{1, t}\left(-\theta_{1}\right)\right]+b_{1, t}\left(-\theta_{1}\right)-b_{2, t}^{*}\left[-\theta_{2}+a_{1}^{*}\left(-\theta_{1}\right)\right]-b_{1, t}^{*}\left(-\theta_{1}\right),
\end{aligned}
$$

with

$$
\begin{aligned}
& b_{1, t}\left(u_{1}\right)=-\nu_{1}^{*^{\prime}} Z_{t} \log \left(1-u_{1} \mu_{1}^{*}\right) \\
& b_{2, t}\left(u_{2}\right)=-\nu_{2}^{*^{\prime}} Z_{t} \log \left(1-u_{2} \mu_{2}^{*}\right),
\end{aligned}
$$

and the risk premium of the digital asset is still given by relation (47).

\section{Derivative Pricing}

\subsection{Generalization of the recursive pricing formula}

In the previous sections, thanks to the feature that the process $\left(\tilde{x}_{t}, z_{t}\right)$ is Car in the risk-neutral world, we have derived explicit recursive formulas for the zero-coupon bond price $B(t, h)$. In fact, as noted in Gourieroux, Monfort and Polimenis (2003), the recursive approach can be generalized to the case of other assets. In particular, given that Car processes have an exponential-affine multihorizon (complex) conditional Laplace transform, we are able to determine explicit or quasi explicit pricing formula for interest rate derivative prices. Let us consider a class of payoffs $g\left(\tilde{X}_{t+h}, Z_{t+h}\right)$, $(t, h)$ varying, for a given $g$ function and let us assume that the price at $t$ of this payoff is of the form:

$$
P_{t}(g, h)=\exp \left[C_{h}(g)^{\prime} \tilde{X}_{t}+D_{h}(g)^{\prime} Z_{t}\right] .
$$


It is clear that:

$$
\begin{aligned}
& \exp \left[C_{h}(g)^{\prime} \tilde{X}_{t}+D_{h}(g)^{\prime} Z_{t}\right] \\
= & E_{t}\left[M_{t, t+1} \exp \left(C_{h-1}(g)^{\prime} \tilde{X}_{t+1}+D_{h-1}(g)^{\prime} Z_{t+1}\right)\right] \\
= & \exp \left(-c^{\prime} \tilde{X}_{t}-d^{\prime} Z_{t}\right) E_{t}^{\mathbb{Q}}\left[\exp \left(C_{h-1}(g)^{\prime} \tilde{X}_{t+1}+D_{h-1}(g)^{\prime} Z_{t+1}\right)\right] ;
\end{aligned}
$$

so the sequences $C_{h}(g), D_{h}(g), h \geq 1$, follow recursive equations which does not depend on $g$ and, therefore, are identical to the case $g=1$, that is to say to the zero-coupon bond pricing formulas given in the previous sections. The only condition for (93) to be true is to hold for $h=1$ and, of course, this initial condition depends on $g$.

Formula (93) is valid for $h=1$ if $g\left(\tilde{X}_{t+h}, Z_{t+h}\right)=\exp \left(\tilde{u}^{\prime} \tilde{X}_{t+h}+\tilde{v}^{\prime} Z_{t+h}\right)$ for some vector $\tilde{u}$ and $\tilde{v}$. Indeed, using the notations

$$
\begin{aligned}
& \tilde{u}^{\prime} \tilde{X}_{t+1}=u_{1}^{\prime} \tilde{x}_{t+1}+u_{-1}^{\prime} \tilde{X}_{t} \\
& \tilde{v}^{\prime} Z_{t+1}=v_{1}^{\prime} z_{t+1}+v_{-1}^{\prime} Z_{t},
\end{aligned}
$$

with $u_{-1}^{\prime}=\left(u_{2}^{\prime}, \ldots, u_{p}^{\prime}, 0\right), v_{-1}^{\prime}=\left(v_{2}^{\prime}, \ldots, v_{p}^{\prime}, 0\right)$, we get:

$$
\begin{array}{r}
P_{t}(\tilde{u}, \tilde{v} ; 1)=\exp \left(-c^{\prime} \tilde{X}_{t}-d^{\prime} Z_{t}+u_{-1}^{\prime} \tilde{X}_{t}+v_{-1}^{\prime} Z_{t}\right) \\
\times E_{t}^{\mathbb{Q}}\left[\exp \left(u_{1}^{\prime} \tilde{x}_{t+1}+v_{1}^{\prime} z_{t+1}\right)\right],
\end{array}
$$

which, using the Car representation of $\left(\tilde{x}_{t+1}, z_{t+1}\right)$ under the probability $\mathbb{Q}$, has obviously the exponential linear form (93) and provides the initial conditions of the recursive equations. The standard recursive equations provide the price $P_{t}(\tilde{u}, \tilde{v} ; h)$ at date $t$ for the payoff $\exp \left(\tilde{u}^{\prime} \tilde{X}_{t+h}+\right.$ $\left.\tilde{v}^{\prime} Z_{t+h}\right)$. So we have the following proposition.

Proposition 16 : The price $P_{t}(\tilde{u}, \tilde{v} ; h)$ at time $t$ of the payoff $g\left(\tilde{X}_{t+h}, Z_{t+h}\right)=\exp \left(\tilde{u}^{\prime} \tilde{X}_{t+h}+\tilde{v}^{\prime} Z_{t+h}\right)$ has the exponential form (93) where $C_{h}(g)$ and $D_{h}(g)$ follow the same recursive equations as in the zero-coupon bond case with initial values $C_{1}(g)$ and $D_{1}(g)$ given by the coefficients of $\tilde{X}_{t}$ and $Z_{t}$ in equation (94).

When $\tilde{u}$ and $\tilde{v}$ have complex components, $P_{t}(\tilde{u}, \tilde{v} ; h)$ provides the complex Laplace transform $E_{t}\left[M_{t, t+h} \exp \left(\tilde{u}^{\prime} \tilde{X}_{t+h}+\tilde{v}^{\prime} Z_{t+h}\right)\right]$.

\subsection{Explicit and quasi explicit pricing formulas}

The explicit formulas for zero-coupon bond prices also immediately provide explicit formulas for some derivatives like swaps. Moreover, the result of Section 5.1, where $\tilde{u}$ and $\tilde{v}$ have complex components, can be used to price payoffs of the form:

$$
\left[\exp \left(\tilde{u}_{1}^{\prime} \tilde{X}_{t+h}+\tilde{v}_{1}^{\prime} Z_{t+h}\right)-\exp \left(\tilde{u}_{2}^{\prime} \tilde{X}_{t+h}+\tilde{v}_{2}^{\prime} Z_{t+h}\right)\right]^{+}
$$

like caps, floors or options on zero-coupon bonds. Let us consider, for instance, the problem to price, at date $t$, a European call option on the zero-coupon bond $B(t+h, H-h)$, then the pricing 
relation is :

$$
\begin{aligned}
p_{t}(K, h) & =E_{t}\left[M_{t, t+h}(B(t+h, H-h)-K)^{+}\right] \\
& =E_{t}\left[M_{t, t+h}(\exp [-(H-h) R(t+h, H-h)]-K)^{+}\right],
\end{aligned}
$$

and, substituting here the yield to maturity formula (68), for the $\operatorname{SVARN}(p)$ model, or formula (91), for the $\operatorname{SVARG}(p)$ model, we can write :

$$
\begin{aligned}
& p_{t}(K, h)=E_{t}\left[M_{t, t+h}\left(\exp \left[C_{H-h}^{\prime} \tilde{X}_{t+h}+D_{H-h}^{\prime} Z_{t+h}\right]-K\right)^{+}\right] \\
& =E_{t}\left[M_{t, t+h}\left(\exp \left[C_{H-h}^{\prime} \tilde{X}_{t+h}+D_{H-h}^{\prime} Z_{t+h}\right]-K\right) \mathbb{I}_{\left[-C_{H-h}^{\prime} \tilde{X}_{t+h}-D_{H-h}^{\prime} Z_{t+h}<-\log K\right]}\right] \\
& =E_{t}\left[M_{t, t+h}\left(\exp \left[C_{H-h}^{\prime} \tilde{X}_{t+h}+D_{H-h}^{\prime} Z_{t+h}\right]\right) \mathbb{I}_{\left[-C_{H-h}^{\prime} \tilde{X}_{t+h}-D_{H-h}^{\prime} Z_{t+h}<-\log K\right]}\right] \\
& \left.-K E_{t}\left[M_{t, t+h} \mathbb{I}_{\left[-C_{H-h}^{\prime}\right.} \tilde{X}_{t+h}-D_{H-h}^{\prime} Z_{t+h}<-\log K\right]\right] \\
& =G_{t}\left(C_{H-h}, D_{H-h},-C_{H-h},-D_{H-h},-\log K ; h\right) \\
& -K G_{t}\left(0,0,-C_{H-h},-D_{H-h},-\log K ; h\right),
\end{aligned}
$$

where $\mathbb{I}$ denotes the indicator function, and where

$$
G_{t}\left(\tilde{u}_{0}, \tilde{v}_{0}, \tilde{u}_{1}, \tilde{v}_{1}, K ; h\right)=E_{t}\left[M_{t, t+h}\left(\exp \left[\tilde{u}_{0}^{\prime} \tilde{X}_{t+h}+\tilde{v}_{0}^{\prime} Z_{t+h}\right]\right) \mathbb{I}_{\left[-\tilde{u}_{1}^{\prime} \tilde{X}_{t+h}-\tilde{v}_{1}^{\prime} Z_{t+h}<K\right]}\right]
$$

denotes the truncated real Laplace transform which we can be deduced from the (untruncated) complex Laplace transform. More precisely, we have the following formula [see Duffie, Pan, Singleton (2000) for details]:

$$
\begin{aligned}
& G_{t}\left(\tilde{u}_{0}, \tilde{v}_{0}, \tilde{u}_{1}, \tilde{v}_{1}, K ; h\right)=\frac{P_{t}\left(\tilde{u}_{0}, \tilde{v}_{0}, h\right)}{2} \\
& \quad-\frac{1}{\pi} \int_{0}^{+\infty}\left[\frac{\operatorname{Im}\left[P_{t}\left(\tilde{u}_{0}+i \tilde{u}_{1} y, \tilde{v}_{0}+i \tilde{v}_{1} y ; h\right)\right] \exp (-i y K)}{y}\right] d y
\end{aligned}
$$

where $\operatorname{Im}(z)$ denotes the imaginary part of the complex number $z$. So, formula (96) is quasi explicit since it only requires a simple (one-dimensional) integration to derive the values of $G_{t}$. 


\section{Conclusions}

This paper has developed a general discrete-time modeling of the term structure of interest rates able to take into account at the same time several important features : a) an historical dynamics of the factor involving several lagged values and switching regimes; b) a specification of the exponential-affine stochastic discount factor (SDF) with time-varying coefficients implying stochastic risk premia, functions of the present and past values of the factor $\left(x_{t}\right)$ and the regime indicator function $\left(z_{t}\right)$; c) explicit or quasi explicit formulas for zero-coupon bond (the Generalized Linear Term Structure formula) and interest rate derivative prices; d) the positivity of the yields at each maturity (in the Autoregressive Gamma framework), regardless the observable or latent nature of the factor $\left(x_{t}\right)$. We have studied, in the Gaussian framework, the theory of $\operatorname{SARN}(p)$ and the $\operatorname{SVARN}(p)$ Factor-Based Term Structure Models, providing a generalization of the recent modelisation proposed by Dai, Singleton and Yang (2006). In the case of an observable factor (yields at different maturities), we have presented a two-step estimation procedure based on a generalization of the Kim's smoothing algorithm. In the Autoregressive Gamma setting, we have proposed the $\operatorname{SARG}(p)$ and the SVARG $(p)$ Factor-Based Term Structure Models, extending several discrete time CIR term structure models like Naik and Lee (1997) and Bansal and Zhou (2002). 


\section{Appendix 1 : Proof of Proposition 3}

$$
\begin{aligned}
& E\left[\exp \left(u_{1} y_{1, t+1}+u_{2} y_{2, t+1}\right) \mid \underline{y_{1, t}}, \underline{y_{2, t}}\right] \\
&= E\left[\exp \left(u_{2} y_{2, t+1}\right) E\left(\exp \left(u_{1} y_{1, t+1}\right) \mid \underline{y_{1, t}}, \underline{y_{2, t+1}}\right) \mid \underline{y_{1, t}}, \underline{y_{2, t}}\right] \\
&= \exp \left[a_{1}\left(u_{1}\right)\left(\beta_{11} y_{1, t}+\beta_{12} y_{2, t}\right)+b_{1}\left(u_{1}\right)\right] E_{t}\left[\exp \left(\left(u_{2}+a_{1}\left(u_{1}\right) \beta_{o}\right) y_{2, t+1}\right) \mid \underline{y_{1, t}}, \underline{y_{2, t}}\right] \\
&=\exp \left[a_{1}\left(u_{1}\right)\left(\beta_{11} y_{1, t}+\beta_{12} y_{2, t}\right)+b_{1}\left(u_{1}\right)\right. \\
&\left.\quad+a_{2}\left(u_{2}+a_{1}\left(u_{1}\right) \beta_{o}\right)\left(\beta_{21} y_{1, t}+\beta_{22} y_{2, t}\right)+b_{2}\left(u_{2}+a_{1}\left(u_{1}\right) \beta_{o}\right)\right] \\
&=\exp \left\{\left[a_{1}\left(u_{1}\right) \beta_{11}+a_{2}\left(u_{2}+a_{1}\left(u_{1}\right) \beta_{o}\right) \beta_{21}\right] y_{1, t}\right. \\
&\left.\quad+\left[a_{1}\left(u_{1}\right) \beta_{12}+a_{2}\left(u_{2}+a_{1}\left(u_{1}\right) \beta_{o}\right) \beta_{22}\right] y_{2, t}+b_{1}\left(u_{1}\right)+b_{2}\left(u_{2}+a_{1}\left(u_{1}\right) \beta_{o}\right)\right\} .
\end{aligned}
$$

\section{Appendix 2 : Proof of Propositions 5 and 6}

Proof of Proposition 5 : Let us first consider an asset providing the payoff $\exp \left(-\theta x_{t+1}\right)$ at $t+1$; the price at $t$ of this asset is

$$
\begin{aligned}
p_{t}= & E_{t}\left[M_{t, t+1} \exp \left(-\theta x_{t+1}\right)\right] \\
= & \exp \left[-r_{t+1}-\theta \nu\left(Z_{t}\right)-\theta \varphi\left(Z_{t}\right)^{\prime} X_{t}-\frac{1}{2} \Gamma\left(Z_{t}, X_{t}\right)^{2}\right] \times \\
\quad & \quad E_{t}\left\{\exp \left[\left[\Gamma\left(Z_{t}, X_{t}\right)-\theta \sigma\left(Z_{t}\right)\right] \varepsilon_{t+1}\right]\right\} \\
= & \exp \left[-r_{t+1}-\theta \nu\left(Z_{t}\right)-\theta \varphi\left(Z_{t}\right)^{\prime} X_{t}-\theta \Gamma\left(Z_{t}, X_{t}\right) \sigma\left(Z_{t}\right)+\frac{\theta^{2}}{2} \sigma^{2}\left(Z_{t}\right)\right],
\end{aligned}
$$

and

$$
\begin{aligned}
E_{t} p_{t+1}= & E_{t}\left[\exp \left(-\theta x_{t+1}\right)\right] \\
= & \exp \left[-\theta \nu\left(Z_{t}\right)-\theta \varphi\left(Z_{t}\right)^{\prime} X_{t}\right] \times \\
& E_{t}\left\{\exp \left[\left[-\theta \sigma\left(Z_{t}\right)\right] \varepsilon_{t+1}\right]\right\} \\
= & \exp \left[-\theta \nu\left(Z_{t}\right)-\theta \varphi\left(Z_{t}\right)^{\prime} X_{t}+\frac{\theta^{2}}{2} \sigma^{2}\left(Z_{t}\right)\right] .
\end{aligned}
$$

Finally, from Definition 4, the risk premium is:

$$
\omega_{t}(\theta)=\theta \Gamma\left(Z_{t}, X_{t}\right) \sigma\left(Z_{t}\right) .
$$

Proof of Proposition 6 : Similarly, if we consider a digital asset providing one money unit at $t+1$ if $z_{t+1}=e_{j}$, we get:

$$
\begin{aligned}
p_{t} & =E_{t}\left[M_{t, t+1} \mathbb{I}_{\left(e_{j}\right)}\left(z_{t+1}\right)\right] \\
& =\exp \left[-r_{t+1}\right] \exp \left[-\delta_{j}\left(Z_{t}, X_{t}\right)\right] \pi\left(z_{t}, e_{j} ; X_{t}\right)
\end{aligned}
$$


and

$$
\begin{aligned}
E_{t} p_{t+1} & =E_{t}\left[\mathbb{I}_{\left(e_{j}\right)}\left(z_{t+1}\right)\right] \\
& =\pi\left(z_{t}, e_{j} ; X_{t}\right) .
\end{aligned}
$$

Therefore, applying Definition 4, the risk premium is :

$$
\omega_{t}(\theta)=\delta_{j}\left(X_{t}, Z_{t}\right) .
$$

\section{Appendix 3 : Proof of Proposition 7}

The Laplace transform of the one-period conditional risk-neutral probability is:

$$
\begin{gathered}
E_{t}^{\mathbb{Q}}\left[\exp \left(u x_{t+1}+v^{\prime} z_{t+1}\right)\right] \\
=E_{t}\left\{\operatorname { e x p } \left[\Gamma\left(Z_{t}, X_{t}\right) \varepsilon_{t+1}-\frac{1}{2} \Gamma\left(Z_{t}, X_{t}\right)^{2}-\delta^{\prime}\left(Z_{t}, X_{t}\right) z_{t+1}\right.\right. \\
\left.\left.+u\left[\nu\left(Z_{t}\right)+\varphi\left(Z_{t}\right)^{\prime} X_{t}+\sigma\left(Z_{t}\right) \varepsilon_{t+1}\right]+v^{\prime} z_{t+1}\right]\right\} \\
=\exp \left\{u\left[\varphi^{\prime}\left(Z_{t}\right) X_{t}+\Gamma\left(Z_{t}, X_{t}\right) \sigma\left(Z_{t}\right)\right]+u \nu\left(Z_{t}\right)+\frac{1}{2} u^{2} \sigma\left(Z_{t}\right)^{2}\right\} \times \\
\sum_{j=1}^{J} \pi\left(z_{t}, e_{j} ; X_{t}\right) \exp \left[\left(v-\delta\left(Z_{t}, X_{t}\right)\right)^{\prime} e_{j}\right] \\
=\exp \left\{u\left[\varphi\left(Z_{t}\right)+\tilde{\gamma}\left(Z_{t}\right) \sigma\left(Z_{t}\right)\right]^{\prime} X_{t}+u\left[\nu\left(Z_{t}\right)+\gamma\left(Z_{t}\right) \sigma\left(Z_{t}\right)\right]+\frac{1}{2} u^{2} \sigma\left(Z_{t}\right)^{2}\right\} \times \\
\sum_{j=1}^{J} \pi\left(z_{t}, e_{j} ; X_{t}\right) \exp \left[\left(v-\delta\left(Z_{t}, X_{t}\right)\right)^{\prime} e_{j}\right] .
\end{gathered}
$$

Therefore, we get the result of Proposition 7 . 


\section{Appendix 4 : Proof of Proposition 8}

Assuming that (52) is true for $h-1$, we get:

$$
\begin{aligned}
B(t, h)= & \exp \left(C_{h}^{\prime} X_{t}+D_{h}^{\prime} Z_{t}\right) \\
= & \exp \left(-r_{t+1}\right) E_{t}^{Q}[B(t+1, h-1)] \\
= & \exp \left[-c^{\prime} X_{t}-d^{\prime} Z_{t}\right] E_{t}^{Q}\left[\exp \left(C_{h-1}^{\prime} X_{t+1}+D_{h-1}^{\prime} Z_{t+1}\right)\right] \\
= & \exp \left[-c^{\prime} X_{t}-d^{\prime} Z_{t}\right] \times \\
& E_{t}^{Q}\left[\exp \left(C_{h-1}^{\prime}\left[\Phi^{*} X_{t}+\left(\nu^{*^{\prime}} Z_{t}+\sigma^{*^{\prime}} Z_{t} \xi_{t+1}\right) e_{1}\right]+D_{1, h-1}^{\prime} z_{t+1}+\tilde{D}_{h-1}^{\prime} Z_{t}\right)\right] \\
= & \exp \left[\left(\Phi^{*^{\prime}} C_{h-1}-c\right)^{\prime} X_{t}+\left(-d+C_{1, h-1} \nu^{*}+\frac{1}{2} C_{1, h-1}^{2} \sigma^{* 2}+\tilde{D}_{h-1}\right)^{\prime} Z_{t}\right] \times \\
= & \quad \exp \left\{\left(E _ { t } ^ { Q } \left[\exp \left(\Phi_{1, h-1}^{\prime *^{\prime}} C_{h-1}-c\right)^{\prime} X_{t+1}+\right.\right.\right. \\
& \left.\quad\left[-d+C_{1, h-1} \nu^{*}+\frac{1}{2} C_{1, h-1}^{2} \sigma^{* 2}+\tilde{D}_{h-1}+F\left(D_{1, h-1}\right)\right]^{\prime} Z_{t}\right\},
\end{aligned}
$$

and the result follows by identification.

\section{Appendix 5 : Proof of Proposition 9}

Using the lag polynomials:

$$
\begin{aligned}
C_{h}(L) & =-\frac{1}{h}\left(C_{1, h}+C_{2, h} L+\ldots+C_{p, h} L^{p-1}\right) \\
D_{h}(L) & =-\frac{1}{h}\left(D_{1, h}+D_{2, h} L+\ldots+D_{p+1, h} L^{p}\right) \\
\Psi\left(L, Z_{t}\right) & =1-\varphi_{1}\left(Z_{t}\right) L-\ldots-\varphi_{p}\left(Z_{t}\right) L^{p},
\end{aligned}
$$

we get from (54):

$$
R(t, h)=C_{h}(L) x_{t}+D_{h}(L)^{\prime} z_{t},
$$

and

$$
\begin{aligned}
\Psi\left(L, Z_{t}\right) R(t+1, h) & =C_{h}(L) \Psi\left(L, Z_{t}\right) x_{t+1}+D_{h}(L) \Psi\left(L, Z_{t}\right) z_{t+1}, \\
& =D_{h}(L) \Psi\left(L, Z_{t}\right) z_{t+1}+C_{h}(L) \nu\left(Z_{t}\right)+C_{h}(L)\left[\left(\sigma^{*^{\prime}} Z_{t}\right) \varepsilon_{t+1}\right] .
\end{aligned}
$$




\section{Appendix 6 : Proof of Proposition 11}

The Laplace transform of the one-period conditional risk-neutral distribution is :

$$
\begin{gathered}
E_{t}^{\mathbb{Q}}\left[\exp \left(u^{\prime} \tilde{x}_{t+1}+v^{\prime} z_{t+1}\right)\right] \\
=E_{t}\left\{\operatorname { e x p } \left[\Gamma\left(Z_{t}, \tilde{X}_{t}\right)^{\prime} \varepsilon_{t+1}-\frac{1}{2} \Gamma\left(Z_{t}, \tilde{X}_{t}\right)^{\prime} \Gamma\left(Z_{t}, \tilde{X}_{t}\right)-\delta^{\prime}\left(Z_{t}, \tilde{X}_{t}\right) z_{t+1}\right.\right. \\
\left.\left.\quad+u^{\prime}\left[\tilde{\nu}\left(Z_{t}\right)+\tilde{\Phi}\left(Z_{t}\right) \tilde{X}_{t}+S\left(Z_{t}\right) \varepsilon_{t+1}\right]+v^{\prime} z_{t+1}\right]\right\} \\
=\exp \left\{u^{\prime}\left[\tilde{\Phi}\left(Z_{t}\right) \tilde{X}_{t}+S\left(Z_{t}\right) \Gamma\left(Z_{t}, \tilde{X}_{t}\right)\right]+u^{\prime} \tilde{\nu}\left(Z_{t}\right)+\frac{1}{2} u^{\prime} S\left(Z_{t}\right) S\left(Z_{t}\right)^{\prime} u\right\} \times \\
\quad \sum_{j=1}^{J} \pi\left(z_{t}, e_{j} ; \tilde{X}_{t}\right) \exp \left[\left(v-\delta\left(Z_{t}, \tilde{X}_{t}\right)\right)^{\prime} e_{j}\right] \\
=\exp \left\{u^{\prime}\left[\tilde{\Phi}\left(Z_{t}\right)+S\left(Z_{t}\right) \tilde{\Gamma}\left(Z_{t}, \tilde{X}_{t}\right)\right] \tilde{X}_{t}+u^{\prime}\left[\tilde{\nu}\left(Z_{t}\right)+S\left(Z_{t}\right) \gamma\left(Z_{t}\right)\right]+\frac{1}{2} u^{\prime} S\left(Z_{t}\right) S\left(Z_{t}\right)^{\prime} u\right\} \times \\
\quad \sum_{j=1}^{J} \pi\left(z_{t}, e_{j} ; \tilde{X}_{t}\right) \exp \left[\left(v-\delta\left(Z_{t}, \tilde{X}_{t}\right)\right)^{\prime} e_{j}\right] .
\end{gathered}
$$

Therefore, we get the result of Proposition 11.

\section{Appendix 7 : Proof of Proposition 12}

Assuming that (66) is true for $h-1$, we get:

$$
\begin{aligned}
& B(t, h)= \exp \left(C_{h}^{\prime} \tilde{X}_{t}+D_{h}^{\prime} Z_{t}\right) \\
&= \exp \left(-r_{t+1}\right) E_{t}^{Q}[B(t+1, h-1)] \\
&= \exp \left[-c^{\prime} \tilde{X}_{t}-d^{\prime} Z_{t}\right] E_{t}^{Q}\left[\exp \left(C_{h-1}^{\prime} \tilde{X}_{t+1}+D_{h-1}^{\prime} Z_{t+1}\right)\right] \\
&= \exp \left[-c^{\prime} \tilde{X}_{t}-d^{\prime} Z_{t}\right] \times \\
& E_{t}^{Q}\left[\operatorname { e x p } \left(C_{h-1}^{\prime} \tilde{\Phi}^{*} \tilde{X}_{t}+C_{1, h-1}\left(\nu_{1}^{*} Z_{t}+S_{1}^{*}\left(Z_{t}\right) \xi_{t+1}\right)\right.\right. \\
&\left.\left.\quad+C_{p+1, h-1}\left(\nu_{2}^{*} Z_{t}+S_{2}^{*}\left(Z_{t}\right) \xi_{t+1}\right)+D_{1, h-1}^{\prime} z_{t+1}+\tilde{D}_{h-1}^{\prime} Z_{t}\right)\right] \\
& \quad \quad+\frac{1}{2} C_{1, h-1}^{2}\left(\sigma_{1}^{* 2}+\varphi_{o}^{* 2} \sigma_{2}^{* 2}\right)+\left(C_{1, h-1}\right)\left(C_{p+1, h-1}\right) \varphi_{o}^{* 2} \sigma_{2}^{* 2} \\
&\left.\left.\quad+\frac{1}{2} C_{p+1, h-1}^{2} \sigma_{2}^{* 2}+\tilde{D}_{h-1}+F\left(D_{1, h-1}\right)\right]^{\prime} Z_{t}\right]
\end{aligned}
$$

and the result follows by identification. 


\section{Appendix 8 : A Generalization of the Kim's Smoothing Algorithm}

The proof of the Smoothing algorithm for the general model:

$$
\begin{aligned}
& y_{t+1}=\vartheta_{y}\left(\underline{y_{t}}, \underline{z_{t-p+1}^{t+1}}, \eta_{t+1}\right) \\
& z_{t+1}=\vartheta_{z}\left(\underline{y_{t}}, z_{t}, \varepsilon_{t+1}\right),
\end{aligned}
$$

with $\left(\varepsilon_{t}\right)$ and $\left(\eta_{t}\right)$ independent white noise processes, $\left(y_{t}\right)$ an observable process, $\left(z_{t}\right)$ a nonhomogeneous (latent) Markov chain, where $\underline{y_{t}}=\left(y_{t}, y_{t-1}, \ldots\right), \underline{z_{t-p+1}^{t+1}}=\left(z_{t-p+1}, \ldots, z_{t+1}\right)$, and where $p \in \mathbb{N}$ and $h \in \mathbb{N}_{+}$, is based on the following three lemmas.

Lemma I : Model (A.1) can be written, for each integer $h \geq 2$, in the following way :

$$
\begin{aligned}
& y_{t+h}=\vartheta_{y}^{(h)}\left(\underline{y_{t}}, \underline{z_{t-p+1}^{t+1}}, \eta_{t+1}, \ldots, \eta_{t+h}, \varepsilon_{t+2}, \ldots, \varepsilon_{t+h}\right) \\
& z_{t+h}=\vartheta_{z}^{(h)}\left(\underline{y_{t}}, \underline{z_{t-p+1}^{t+1}}, \eta_{t+1}, \ldots, \eta_{t+h-1}, \varepsilon_{t+2}, \ldots, \varepsilon_{t+h}\right),
\end{aligned}
$$

[Proof : by recursive substitution, starting from $\left.\left(y_{t+1}, z_{t+1}\right)\right]$. In particular, for each $h \geq 1$ and replacing $t$ by $t+p$, we have:

$$
\begin{aligned}
& y_{t+p+h}=\vartheta_{y}^{(h)}\left(\underline{y_{t+p}}, \underline{z_{t+1}^{t+p+1}}, \eta_{t+p+1}, \ldots, \eta_{t+p+h}, \varepsilon_{t+p+2}, \ldots, \varepsilon_{t+p+h}\right) \\
& z_{t+p+h}=\vartheta_{z}^{(h)}\left(\underline{y_{t+p}}, \underline{z_{t+1}^{t+p+1}}, \eta_{t+p+1}, \ldots, \eta_{t+p+h-1}, \varepsilon_{t+p+2}, \ldots, \varepsilon_{t+p+h}\right),
\end{aligned}
$$

where, with the notation $I_{\varepsilon}(t+p, h):=\left(\varepsilon_{t+p+2}, \ldots, \varepsilon_{t+p+h}\right)$, we assume $I_{\varepsilon}(t+p, 1)=\emptyset$.

Lemma II : If $I_{1} \subset I \subset I_{2}$ and $\mathbb{P}\left[z_{t} \mid I_{1}\right]=\mathbb{P}\left[z_{t} \mid I_{2}\right]$, then $\mathbb{P}\left[z_{t} \mid I_{1}\right]=\mathbb{P}\left[z_{t} \mid I\right]=\mathbb{P}\left[z_{t} \mid I_{2}\right]$ [Proof : straightforward].

Lemma III : Given model (A.1), the following relation holds :

$$
\mathbb{P}\left[z_{t} \mid \underline{z_{t+1}^{t+p+1}}, \underline{y_{T}}\right]=\mathbb{P}\left[z_{t} \mid \underline{z_{t+1}^{t+p+1}}, \underline{y_{t+p}}\right] .
$$

Proof : Given the three sets $I_{1}=\left(\underline{z_{t+1}^{t+p+1}}, \underline{y_{t+p}}\right), I=\left(\underline{z_{t+1}^{t+p+1}}, \underline{y_{T}}\right)$ and $I_{2}=\left(\underline{z_{t+1}^{t+p+1}}, \underline{y_{t+p}}, \underline{\eta_{t+p+1}^{T}}\right.$, $\left.\underline{\varepsilon_{t+p+2}^{T}}\right)$, we have that :

(i) $I_{1} \subset I$,

(ii) $I \subset I_{2}$,

(iii) $\left.\underline{\left(\eta_{t+p+1}^{T},\right.} \underline{\varepsilon_{t+p+2}^{T}}\right) \perp\left(\underline{z_{t+1}^{t+p+1}}, \underline{y_{t+p}}, z_{t}\right)$.

The proof of relation $(i)$ is straightforward. Relation (ii) holds given that, from Lemma I, we can always write, for any $s>t+p$ :

$$
y_{s}=\vartheta_{y}^{(s-t-p)}\left(\underline{y_{t+p}}, \underline{z_{t+1}^{t+p+1}}, \eta_{t+p+1}, \ldots, \eta_{s}, \varepsilon_{t+p+2}, \ldots, \varepsilon_{s}\right),
$$


and, therefore, $\left(\underline{y_{T}}\right) \subset\left(\underline{y_{t+p}}, \underline{z_{t+1}^{t+p+1}}, \underline{\eta_{t+p+1}^{T}}, \underline{\varepsilon_{t+p+2}^{T}}\right)$, that is, $I \subset I_{2}$.

With regard to relation (iii), from Lemma I applied to $\left(z_{t+1}, y_{t+1}\right)$, we have:

$$
\begin{aligned}
& z_{t+p+1}=\vartheta_{z}^{(t+p+1)}\left(\underline{y_{0}}, \underline{z_{-p+1}^{1}}, \eta_{1}, \ldots, \eta_{t+p}, \varepsilon_{2}, \ldots, \varepsilon_{t+p+1}\right) \\
& y_{t+p}=\vartheta_{y}^{(t+p)}\left(\underline{y_{0}}, \underline{z_{-p+1}^{1}}, \eta_{1}, \ldots, \eta_{t+p}, \varepsilon_{2}, \ldots, \varepsilon_{t+p}\right)
\end{aligned}
$$

and given that $z_{t}=\vartheta_{z}\left(\underline{y_{t-1}}, z_{t-1}, \varepsilon_{t}\right)$, we conclude (using the notation $\mathbb{P}$ for the p.d.f.) :

$$
\mathbb{P}\left(\underline{\eta_{t+p+1}^{T}}, \underline{\varepsilon_{t+p+2}^{T}} \mid \underline{z_{t+1}^{t+p+1}}, \underline{y_{t+p}}, z_{t}\right)=\mathbb{P}\left(\underline{\eta_{t+p+1}^{T}}, \underline{\varepsilon_{t+p+2}^{T}}\right)
$$

and relation (iii) is proved. Now, given property (iii), we have:

$$
\begin{aligned}
\mathbb{P}\left[z_{t} \mid I_{2}\right] & =\frac{\mathbb{P}\left[z_{t}, I_{2}\right]}{\mathbb{P}\left[I_{2}\right]} \\
& =\frac{\mathbb{P}\left[\underline{z_{t+1}^{t+p+1}}, \underline{y_{t+p}}, z_{t}, \underline{\eta_{t+p+1}^{T}}, \underline{\left.\varepsilon_{t+p+2}^{T}\right]}\right.}{\mathbb{P}\left[z_{t+1}^{t+p+1}, \underline{y_{t+p}}, \underline{\eta_{t+p+1}^{T},}, \underline{\left.\varepsilon_{t+p+2}^{T}\right]}\right.} \\
& =\frac{\mathbb{P}\left[\underline{z_{t+1}^{t+p+1}}, \underline{y_{t+p}}, z_{t}\right] \mathbb{P}\left[\underline{\eta_{t+p+1}^{T}}, \underline{\varepsilon_{t+p+2}^{T}}\right]}{\mathbb{P}\left[z_{t+1}^{t+p+1}, \underline{y_{t+p}}\right]} \underline{\mathbb{P}\left[\underline{\eta_{t+p+1}^{T}}, \underline{\left.\varepsilon_{t+p+2}^{T}\right]}\right.} \\
& =\frac{\mathbb{P}\left[z_{t}, I_{1}\right]}{\mathbb{P}\left[I_{1}\right]}=\mathbb{P}\left[z_{t} \mid I_{1}\right],
\end{aligned}
$$

and applying Lemma II, we prove (A.4).

If $p \geq 1$, the smoothing formula is :

$$
\mathbb{P}\left[z_{t}, \ldots, z_{t+p} \mid \underline{y_{T}}\right]=\frac{\mathbb{P}\left[z_{t}, \ldots, z_{t+p} \mid \underline{y_{t+p}}\right]}{\mathbb{P}\left[z_{t+1}, \ldots, z_{t+p} \mid \underline{y_{t+p}}\right]} \sum_{z_{t+p+1}} \mathbb{P}\left[z_{t+1}, \ldots, z_{t+p+1} \mid \underline{y_{T}}\right]
$$

Proof : Applying Lemma III, we can write

$$
\begin{aligned}
& \mathbb{P}\left[z_{t}, \ldots, z_{t+p+1} \mid \underline{y_{T}}\right] \\
= & \mathbb{P}\left[z_{t} \mid z_{t+1}, \ldots, z_{t+p+1}, \underline{y_{T}}\right] \mathbb{P}\left[z_{t+1}, \ldots, z_{t+p+1} \mid \underline{y_{T}}\right] \\
= & \mathbb{P}\left[z_{t} \mid z_{t+1}, \ldots, z_{t+p+1}, \underline{y_{t+p}}\right] \mathbb{P}\left[z_{t+1}, \ldots, z_{t+p+1} \mid \underline{y_{T}}\right] \\
= & \mathbb{P}\left[z_{t+1}, \ldots, z_{t+p+1} \mid \underline{y_{T}}\right] \frac{\mathbb{P}\left[z_{t}, \ldots, z_{t+p+1}, \underline{y_{t+p}}\right]}{\mathbb{P}\left[z_{t+1}, \ldots, z_{t+p+1}, \underline{y_{t+p}}\right]} \\
= & \mathbb{P}\left[z_{t+1}, \ldots, z_{t+p+1} \mid \underline{y_{T}}\right] \frac{\mathbb{P}\left[z_{t+p+1} \mid z_{t+p}, \ldots, z_{t}, \underline{y_{t+p}}\right] \mathbb{P}\left[z_{t+p}, \ldots, z_{t} \mid \underline{y_{t+p}}\right]}{\mathbb{P}\left[z_{t+p+1} \mid z_{t+p}, \ldots, z_{t+1}, \underline{y_{t+p}}\right] \mathbb{P}\left[z_{t+p}, \ldots, z_{t+1} \underline{y_{t+p}}\right]}
\end{aligned}
$$


and, $\left(z_{t}\right)$ being (conditionally) a Markov chain, relation (A.10) can be written:

$$
\begin{aligned}
& \mathbb{P}\left[z_{t}, \ldots, z_{t+p+1} \mid \underline{y_{T}}\right] \\
= & \mathbb{P}\left[z_{t+1}, \ldots, z_{t+p+1} \mid \underline{y_{T}}\right] \frac{\mathbb{P}\left[z_{t}, \ldots, z_{t+p} \mid \underline{y_{t+p}}\right]}{\mathbb{P}\left[z_{t+1}, \ldots, z_{t+p} \mid \underline{y_{t+p}}\right]}
\end{aligned}
$$

now, if we integrate out $z_{t+p+1}$ on the LHS and RHS of (A.11), we obtain (A.9). The smoothing algorithm start, at $t=T-p-1$, from $\mathbb{P}\left[z_{T-p}, \ldots, z_{T-1} \mid \underline{y_{T}}\right]=\sum_{z_{T}} \mathbb{P}\left[z_{T-p}, \ldots, z_{T} \mid \underline{y_{T}}\right]$, with $\mathbb{P}\left[z_{T-p}, \ldots, z_{T} \mid \underline{y_{T}}\right]$ provided by the Kitagawa-Hamilton filter.

If $p=0$, the smoothing formula is :

$$
\mathbb{P}\left[z_{t} \mid \underline{y_{T}}\right]=\mathbb{P}\left[z_{t} \mid \underline{y_{t}}\right] \sum_{z_{t+1}} \frac{\mathbb{P}\left[z_{t+1} \mid z_{t}, \underline{y_{t}}\right] \mathbb{P}\left[z_{t+1} \mid \underline{y_{T}}\right]}{\mathbb{P}\left[z_{t+1} \mid \underline{y_{t}}\right]} .
$$

Proof : Given that

$$
\begin{aligned}
& \mathbb{P}\left[z_{t}, z_{t+1} \mid \underline{y_{T}}\right] \\
= & \mathbb{P}\left[z_{t} \mid z_{t+1}, \underline{y_{T}}\right] \mathbb{P}\left[z_{t+1} \mid \underline{y_{T}}\right] \\
= & \mathbb{P}\left[z_{t} \mid z_{t+1}, \underline{y_{t}}\right] \mathbb{P}\left[z_{t+1} \mid \underline{y_{T}}\right] \\
= & \mathbb{P}\left[z_{t+1} \mid \underline{y_{T}}\right] \frac{\mathbb{P}\left[z_{t}, z_{t+1} \mid \underline{y_{t}}\right]}{\mathbb{P}\left[z_{t+1} \mid \underline{y_{t}}\right]} \\
= & \mathbb{P}\left[z_{t+1} \mid \underline{y_{T}}\right] \frac{\mathbb{P}\left[z_{t+1} \mid z_{t}, \underline{y_{t}}\right] \mathbb{P}\left[z_{t} \mid \underline{y_{t}}\right]}{\mathbb{P}\left[z_{t+1} \mid \underline{y_{t}}\right]}
\end{aligned}
$$

if we integrate out $z_{t+1}$ from the LHS and RHS of (A.13) we obtain (A.12). 
Appendix 9 : Proof of Lemma 1

$$
\begin{aligned}
\tilde{a}(u+\alpha ; \rho, \mu)-\tilde{a}(\alpha ; \rho, \mu) & =\frac{\rho(u+\alpha)}{1-(u+\alpha) \mu}-\frac{\rho \alpha}{1-\alpha \mu} \\
& =\rho \frac{u}{(1-\alpha \mu)^{2}-u \mu(1-\alpha \mu)} \\
& =\frac{\rho}{(1-\alpha \mu)^{2}} \frac{u}{1-\frac{u \mu}{1-\alpha \mu}} \\
& =\frac{\rho^{*} u}{1-u \mu^{*}}=\tilde{a}\left(u ; \rho^{*}, \mu^{*}\right) ; \\
\tilde{b}(u+\alpha ; \nu, \mu)-\tilde{b}(\alpha ; \nu, \mu) & =-\nu \log (1-(u+\alpha) \mu)+-\nu \log (1-\alpha \mu) \\
& =-\nu \log \left[\frac{1-(u+\alpha) \mu}{1-\alpha \mu}\right] \\
& =-\nu \log \left[1-\frac{u \mu}{1-\alpha \mu}\right] \\
& =-\nu \log \left(1-u \mu^{*}\right) \\
& =\tilde{b}\left(u ; \nu, \mu^{*}\right) .
\end{aligned}
$$

\section{Appendix 10 : Proof of Proposition 13}

Assuming that (81) is true for $h-1$, we get:

$$
\begin{aligned}
& B(t, h)= \exp \left(C_{h}^{\prime} X_{t}+D_{h}^{\prime} Z_{t}\right) \\
&= \exp \left[-c^{\prime} X_{t}-d^{\prime} Z_{t}\right] E_{t}^{Q}\left[\exp \left(C_{h-1}^{\prime} X_{t+1}+D_{h-1}^{\prime} Z_{t+1}\right)\right] \\
&= \exp \left(-c^{\prime} X_{t}-d^{\prime} Z_{t}+\tilde{C}_{h-1}^{\prime} X_{t}+\tilde{D}_{h-1}^{\prime} Z_{t}\right) \\
& E_{t}^{Q}\left[\exp \left(C_{1, h-1} x_{t+1}+D_{1, h-1}^{\prime} z_{t+1}\right)\right] \\
&= \exp \left[-c^{\prime} X_{t}-d^{\prime} Z_{t}+\tilde{C}_{h-1}^{\prime} X_{t}+\tilde{D}_{h-1}^{\prime} Z_{t}+A^{*}\left(C_{1, h-1}\right)^{\prime} X_{t}\right. \\
&\left.\quad-\nu^{*^{\prime}} Z_{t} \log \left(1-C_{1, h-1} \mu^{*}\right)+F^{\prime}\left(D_{1, h-1}\right) Z_{t}\right],
\end{aligned}
$$

and the result follows by identification. 


\section{Appendix 11 : Proof of Proposition 14}

The joint conditional Laplace transform of $\left(x_{1, t+1}, x_{2, t+1}\right)$ in the risk-neutral world is:

$$
\begin{gathered}
E_{t}^{\mathbb{Q}}\left[\exp \left(u_{1} x_{1, t+1}+u_{2} x_{2, t+1}\right) \mid \underline{x_{1, t}}, \underline{x_{2, t}}, \underline{z_{t}}\right] \\
=\exp \left\{A_{2, t}\left[u_{2}+\Gamma_{2 t}+a_{1, t}\left(u_{1}+\Gamma_{1 t}\right)\right]^{\prime} \tilde{X}_{t}+b_{2, t}\left(u_{2}+\Gamma_{2 t}+a_{1, t}\left(u_{1}+\Gamma_{1 t}\right)\right)\right. \\
+A_{1, t}\left(u_{1}+\Gamma_{1 t}\right)^{\prime} \tilde{X}_{t}+b_{1, t}\left(u_{1}+\Gamma_{1 t}\right) \\
-A_{2, t}\left(\Gamma_{2 t}+a_{1, t}\left(\Gamma_{1 t}\right)\right)^{\prime} \tilde{X}_{t}-b_{2, t}\left(\Gamma_{2 t}+a_{1, t}\left(\Gamma_{1 t}\right)\right) \\
\left.-A_{1, t}\left(\Gamma_{1 t}\right)^{\prime} \tilde{X}_{t}-b_{1, t}\left(\Gamma_{1 t}\right)\right\} .
\end{gathered}
$$

Using Lemma 2 we get:

$A_{2, t}\left[u_{2}+\Gamma_{2 t}+a_{1, t}\left(u_{1}+\Gamma_{1 t}\right)\right]-A_{2, t}\left(\Gamma_{2 t}+a_{1, t}\left(\Gamma_{1 t}\right)\right)=A\left[u_{2}+a_{1, t}\left(u_{1}+\Gamma_{1 t}\right)-a_{1, t}\left(\Gamma_{1 t}\right) ; \varphi_{2 t}^{*}, \mu_{2 t}^{*}\right]$, with

$$
\varphi_{2 t}^{*}=\frac{\varphi_{2 t}}{\left\{1-\left[\Gamma_{2 t}+a_{1, t}\left(\Gamma_{1 t}\right)\right] \mu_{2 t}\right\}^{2}}, \mu_{2 t}^{*}=\frac{\mu_{2 t}}{\left\{1-\left[\Gamma_{2 t}+a_{1, t}\left(\Gamma_{1 t}\right)\right] \mu_{2 t}\right\}},
$$

and using Lemma 1

$$
\begin{aligned}
& A\left[u_{2}+a_{1, t}\left(u_{1}+\Gamma_{1 t}\right)-a_{1, t}\left(\Gamma_{1 t}\right) ; \varphi_{2 t}^{*}, \mu_{2 t}^{*}\right] \\
= & A\left[u_{2}+\tilde{a}\left(u_{1}+\Gamma_{1 t} ; \varphi_{o t}, \mu_{1, t}\right)-\tilde{a}\left(\Gamma_{1 t} ; \varphi_{o t}, \mu_{1, t}\right) ; \varphi_{2 t}^{*}, \mu_{2 t}^{*}\right] \\
= & A\left[u_{2}+\tilde{a}\left(u_{1} ; \varphi_{o t}^{*}, \mu_{1, t}^{*}\right) ; \varphi_{2 t}^{*}, \mu_{2 t}^{*}\right] \\
= & A_{2, t}^{*}\left[u_{2}+a_{1, t}^{*}\left(u_{1}\right)\right] \text { (say) }
\end{aligned}
$$

with

$$
\varphi_{o t}^{*}=\frac{\varphi_{o t}}{\left(1-\Gamma_{1 t} \mu_{1 t}\right)^{2}}, \mu_{1 t}^{*}=\frac{\mu_{1 t}}{\left(1-\Gamma_{1 t} \mu_{1 t}\right)}
$$

Similarly, we get:

$$
\begin{aligned}
& b_{2, t}\left[u_{2}+\Gamma_{2 t}+a_{1, t}\left(u_{1}+\Gamma_{1 t}\right)\right]-b_{2, t}\left(\Gamma_{2 t}+a_{1, t}\left(\Gamma_{1 t}\right)\right) \\
= & \tilde{b}\left[u_{2}+\tilde{a}\left(u_{1} ; \varphi_{o t}^{*}, \mu_{1, t}^{*}\right) ; \nu_{2 t}^{*}, \mu_{2 t}^{*}\right]=b_{2, t}^{*}\left[u_{2}+a_{1 t}^{*}\left(u_{1}\right)\right] \text { (say), } \\
& b_{1, t}\left(u_{1}+\Gamma_{1 t}\right)-b_{1, t}\left(\Gamma_{1 t}\right)=\tilde{b}_{1}\left(u_{1} ; \nu_{1 t}^{*}, \mu_{1 t}^{*}\right)=b_{1, t}^{*}\left(u_{1}\right) \text { (say), } \\
& A_{1, t}\left(u_{1}+\Gamma_{1 t}\right)-A_{1, t}\left(\Gamma_{1 t}\right)=A_{1}\left(u_{1} ; \varphi_{1 t}^{*}, \mu_{1 t}^{*}\right)=A_{1, t}^{*}\left(u_{1}\right) \text { (say), }
\end{aligned}
$$

with

$$
\varphi_{1 t}^{*}=\frac{\varphi_{1 t}}{\left(1-\Gamma_{1 t} \mu_{1 t}\right)^{2}} .
$$


And finally, the joint conditional Laplace transform of $\left(x_{1, t+1}, x_{2, t+1}\right)$ becomes:

$$
\begin{aligned}
E_{t}^{\mathbb{Q}}\left[\exp \left(u_{1} x_{1, t+1}+u_{2} x_{2, t+1}\right) \mid \underline{x_{1 t}}, \underline{x_{2 t}}, \underline{z_{t}}\right]= & \exp \left\{\left[A_{1, t}^{*}\left(u_{1}\right)+A_{2, t}^{*}\left[u_{2}+a_{1, t}^{*}\left(u_{1}\right)\right]\right]^{\prime} \tilde{X}_{t}\right. \\
& \left.+b_{2, t}^{*}\left[u_{2}+a_{1, t}^{*}\left(u_{1}\right)\right]+b_{1, t}^{*}\left(u_{1}\right)\right\},
\end{aligned}
$$

and the result of Proposition 14 is proved.

\section{Appendix 12 : Proof of Proposition 15}

Assuming that (91) is true for $h-1$, we get:

$$
\begin{aligned}
& B(t, h)=\exp \left(C_{h}^{\prime} \tilde{X}_{t}+D_{h}^{\prime} Z_{t}\right) \\
& =\exp \left[-c^{\prime} \tilde{X}_{t}-d^{\prime} Z_{t}\right] E_{t}^{Q}\left[\exp \left(C_{h-1}^{\prime} \tilde{X}_{t+1}+D_{h-1}^{\prime} Z_{t+1}\right)\right] \\
& =\exp \left(-c^{\prime} \tilde{X}_{t}-d^{\prime} Z_{t}+\tilde{C}_{h-1}^{\prime} \tilde{X}_{t}+\tilde{D}_{h-1}^{\prime} Z_{t}\right) \\
& E_{t}^{Q}\left[\exp \left(C_{1, h-1}^{\prime} x_{1, t+1}+C_{p+1, h-1}^{\prime} x_{2, t+1}+D_{1, h-1}^{\prime} z_{t+1}\right)\right] \\
& =\exp \left[-c^{\prime} \tilde{X}_{t}-d^{\prime} Z_{t}+\tilde{C}_{h-1}^{\prime} \tilde{X}_{t}+\tilde{D}_{h-1}^{\prime} Z_{t}+A_{1}^{*}\left(C_{1, h-1}\right)^{\prime} \tilde{X}_{t}\right. \\
& -\nu_{1}^{*^{\prime}} Z_{t} \log \left(1-C_{1, h-1} \mu_{1}^{*}\right)+A_{2}^{*}\left[C_{p+1, h-1}+a_{1}^{*}\left(C_{1, h-1}\right)\right]^{\prime} \tilde{X}_{t} \\
& \left.-\nu_{2}^{*^{\prime}} Z_{t} \log \left[1-\left(C_{p+1, h-1}+a_{1}^{*}\left(C_{1, h-1}\right)\right) \mu_{2}^{*}\right]+F^{\prime}\left(D_{1, h-1}\right) Z_{t}\right],
\end{aligned}
$$

and the result follows by identification. 


\section{R E F E R E N C E S}

Ahn, D., Dittmar, R., and R. Gallant (2002) : "Quadratic Term Structure Models : Theory and Evidence", Review of Financial Studies, 15, 243-288.

Ait-Sahalia, Y., (1996) : "Testing Continuous-Time Models of the Spot Interest Rate", The Review of Financial Studies, 9, 385-426.

Ang, A., and G. Bekaert, (2002a) : "Regime Switches in Interest Rates", Journal of Business and Economic Statistics, 20, 163-182.

Ang, A., and G. Bekaert, (2002b) : "Short Rate Nonlinearities and Regime Switches", Journal of Economic Dynamics and Control, 26, 1243-1274.

Ang, A., and G. Bekaert, (2005) : "Term Structure of Real Rates and Expected Inflation", Working Paper.

Ang, A., and M. Piazzesi, (2003) : "A No-Arbitrage Vector Autoregression of Term Structure Dynamics with Macroeconomic and Latent Variables", Journal of Monetary Economics, 50, 745787.

Ang, A., Piazzesi, M., and M. Wei, (2006) : "What does the Yield Curve tell us about GDP Growth", Journal of Econometrics, 131, 359-403.

Bansal, R., and H. Zhou, (2002) : "Term Structure of Interest Rates with Regime Shifts", Journal of Finance, 57, 1997-2043.

Beaglehole, D. R., and M. S. Tenney (1991) : "General Solutions of Some Interest Rate Contingent Claim Pricing Equations", Journal of Fixed Income, September, 69-83.

Billio, M., and A. Monfort, (1998) : "Switching State-Space Models Likelihood Function, Filtering and Smoothing", Journal of Statistical Planning and Inference", 68, 65-103.

Boudoukh, J., Richardson, M., Smith, T., and R. F. Whitelaw (1999) : "Regime Shifts and Bond Returns", Working Paper.

Cai, J., (1994) : "A Markov Model of Unconditional Variance in ARCH", Journal of Business and Economic Statistics, 12, 309-316.

Campbell, J., and R. J. Shiller (1991) : "Yield Spreads and Interest Rate Movements : A Bird's Eye View", Review of Economic Studies, 58, 495-514.

Chen, R.-R., and L. Scott, (1993) : "Maximum Likelihood Estimation for a Multifactor Equilibrium Model of the Term Structure of Interest Rates", Journal of Fixed Income, 3, 14-31.

Cheng, P., and O. Scaillet, (2005) : "Linear-Quadratic Jump-Diffusion modeling with Application to Stochastic Volatility", Working Paper.

Cheridito, R., Filipovic, D., and R. Kimmel (2005) : "Market Prices of Risk in Affine Models: Theory and Evidence", forthcoming Journal of Financial Economics. 
Christiansen, C., (2004) : "Regime Switching in the Yield Curve", Journal of Futures Markets, 24, 4, 315-336.

Christiansen, C., and J. Lund (2005) : "Revisiting the Shape of the Yield Curve : the Effect of Interest Rate Volatility", Working Paper.

Cochrane, J., and M. Piazzesi, (2005) : "Bond Risk Premia", American Economic Review, $95 / 1,138-160$.

Collin-Dufresne, P., Goldstein, R. S., and C.S. Jones, (2005) : "Can Interest Rate Volatility be Extracted from the Cross Section of Bond Yields? An Investigation of Unspanned Stochastic Volatility", Working Paper.

Cox, J.C., Ingersoll, J.E. and S. A. Ross (1985) : "A theory of the term structure of interest rates", Econometrica, 53(2), pp. 385-407.

Dai, Q., and K. Singleton (2000) : "Specification Analysis of Affine Term Structure Models", Journal of Finance, 55, 1943-1978.

Dai, Q., and K. Singleton (2002) : "Expectations Puzzles, Time-varying Risk Premia, and Affine Model of the Term Structure", Journal of Financial Economics, 63, 415-441.

Dai, Q., and K. Singleton (2003) : "Term Structure Dynamics in Theory and Reality", Review of Financial Studies, 16, 631-678.

Dai, Q., Singleton, K., and W. Yang (2006) : "Regime Shifts in a Dynamic Term Structure Model of U.S. Treasury Bond Yields", Working Paper.

Dai, Q., Le, A., and K. Singleton (2006) : "Discrete-time Term Structure Models with Generalized Market Prices of Risk", Working Paper.

Darolles, S., Gourieroux, C., and J. Jasiak, (2006) : "Structural Laplace Transform and Compound Autoregressive Models", Journal of Time Series Analysis, 24(4), 477-503.

Driffill, J., and M. Sola, (1994) : "Testing the Term Structure of Interest Rates using a Stationary Vector Autoregression with Regime Switching", Journal of Economic Dynamics and Control, 18, 601-628.

Driffill, J., T., Kenc, and M. Sola, (2003) : "An Empirical Examination of Term Structure Models with Regime Shifts", Working Paper.

Duarte, J. (2004) : "Evaluating An Alternative Risk Preference in Affine Term Structure Models", Review of Financial Studies, 17, 379-404.

Duffee, G. R., (2002) : "Term Premia and Interest Rate Forecasts in Affine Models", Journal of Finance, 57, 405-443.

Duffie, D., Filipovic, D. and W. Schachermayer, (2003) : "Affine processes and applications in finance", The Annals of Applied Probability 13, 984-1053. 
Duffie, D., and R., Kan (1996) : "A yield-factor model of interest rates", Mathematical Finance, $6,379-406$.

Duffie, D., J. Pan, and K. Singleton (2000) : "Transform Analysis and Asset Pricing for Affine Jump Diffusions", Econometrica, 68, 1343-1376.

Evans, M. (2003) : "Real Risk, Inflation Risk, and the Term Structure", The Economic Journal, $113,345-389$.

Garcia, R. and P. Perron (1996) : "An Analysis of Real Interest Rate Under Regime Shifts", Reviews of Economics and Statistics, 1, 111-125.

Gourieroux, C., and J. Jasiak, (2006) : "Autoregressive Gamma Processes", Journal of Forecasting, 25(2), 129-152.

Gourieroux, C., Jasiak, J., and R. Sufana (2004) : "A Dynamic Model for Multivariate Stochastic Volatility : The Wishart Autoregressive Process", Working Paper.

Gourieroux, C., and A. Monfort (2006a) : "Econometric Specifications of Stochastic Discount Factor Models", forthcoming Journal of Econometrics.

Gourieroux, C., and A. Monfort (2006b) : "Domain Restrictions on Interest Rates Implied by No Arbitrage", Crest DP.

Gourieroux, C., Monfort, A. and V. Polimenis (2003) : "Discrete Time Affine Term Structure Models", Crest DP.

Gourieroux, C., Monfort, A. and V. Polimenis (2006) : "Affine Model for Credit Risk Analysis", Journal of Financial Econometrics, 4, 3, 494-530.

Gourieroux, C., and R. Sufana, (2003) : "Wishart Quadratic Term Structure Models", Working Paper.

Gourieroux, C., and R. Sufana, (2006) : "A Classification of Two-Factor Affine Diffusion Term Structure Models", Journal of Financial Econometrics, 4(1), 31-52.

Gray, S., (1996) : "Modeling the Conditional Distribution of Interest Rates as a Regime Switching Process", Journal of Financial Economics, 42, 27-62.

Hamilton, J. D., (1988) : "Rational-Expectations Econometric Analysis of Changes in Regimes - An Investigation of the Term Structure of Interest Rates", Journal of Economic Dynamics and Control, 12, 385-423.

Hamilton, J. D., (1994) : "Time Series Analysis", Princeton University Press, Princeton, New Jersey (USA).

Kim, C. J., (1994) : "Dynamic Linear Models with Markov Switching", Journal of Econometrics, $60,1-22$.

Leippold, M., and L. Wu, (2002) : "Asset Pricing Under the Quadratic Class", Journal of Financial and Quantitative Analysis, 37(2), 271-295. 
Longstaff, F.A., and E.S. Schwartz, (1992) : "Interest rate volatility and the term structure: a two-factor general equilibrium model", Journal of Finance, 47, 1259-1282.

Monfort, A., and F. Pegoraro, (2006) : "Multi-Lag Term Structure Models with Stochastic Risk Premia", CREST DP.

Naik, V., and M. H. Lee (1997) : "Yield Curve Dynamics with Discrete Shifts in Economic Regimes : Theory and Estimation", Working Paper, University of British Columbia.

Pearson, N. D., and T.-S. Sun, (1994) : "Exploiting the Conditional Density in Estimating the Term Structure : An Aplication to the Cox, Ingersoll and Ross Model", Journal of Finance, 49, 1279-1304.

Pegoraro, F. (2006) : "Discrete Time Factor Models for Asset Pricing", PhD Thesis, Université Paris-Dauphine (France).

Piazzesi, M. (2003) : "Affine Term Structure Models", forthcoming Handbook of Financial Econometrics.

Polimenis, V. (2001) : "Essays in Discrete Time Asset Pricing", Ph. D. Thesis, Wharton School, University of Pennsylvania.

Roberds, W., and C. H. Whiteman, (1999) : "Endogenous Term Premia and Anomalies in the Term Structure of Interest Rates : Explaining the Predictability Smile", Journal of Monetary Economics, 44, 555-580.

Stanton, R., (1997) : "A Nonparametric Model of the Term Structure Dynamics and the Market Price of Interest Rate Risk", The Journal of Finance, 52, 1973-2002.

Vasicek, O. (1977) : "An Equilibrium Characterization of the Term Structure", Journal of Financial Economics, 5, 177-188.

Wu, S., and Y. Zeng, (2005) : "A General Equilibrium Model of the Term Structure of Interest Rates under Regime-Switching Risk", International Journal of Theoretical and Applied Finance, $8,839-869$. 